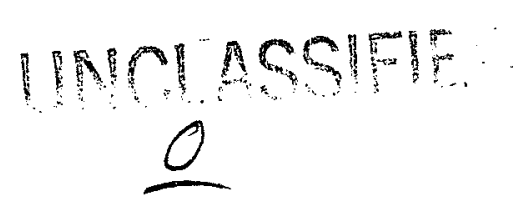

Nevis Cyclotron Laboratories

Columbia University

Department of Physics

New York, New York

\title{
THEORY OF MULTIPLE COULOMB SCATTERING \\ FROM EXTENDED NUCLEI
}

Leon N. Cooper and James Riainwater

CU-70-54-ONR-110-1-Physics

August, 1954

\begin{abstract}
Joint ONR-AEC Program
Office of Naval Research Contract

Contract N6-ori-110-Task No. 1
\end{abstract}

This report has been photostated to fill your request as our supply of copies was exhausted. If you should find that you do not need to retain this copy permanently in your files, we would greatly appreciate your returning it to TIS so that it may be used to fill future requests from other AEC installations.

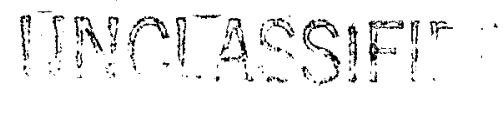





\section{DISCLAIMER}

This report was prepared as an account of work sponsored by an agency of the United States Government. Neither the United States Government nor any agency Thereof, nor any of their employees, makes any warranty, express or implied, or assumes any legal liability or responsibility for the accuracy, completeness, or usefulness of any information, apparatus, product, or process disclosed, or represents that its use would not infringe privately owned rights. Reference herein to any specific commercial product, process, or service by trade name, trademark, manufacturer, or otherwise does not necessarily constitute or imply its endorsement, recommendation, or favoring by the United States Government or any agency thereof. The views and opinions of authors expressed herein do not necessarily state or reflect those of the United States Government or any agency thereof. 


\section{DISCLAIMER}

Portions of this document may be illegible in electronic image products. Images are produced from the best available original document. 
Theory of Multiple Coulomb scattering from Extended Nucle1*

Leon N. Cooper $* * ;$ and James Rainwater

Department of Physics, Columbia University

New York, New York

August, 1954

$*$

This research was supported in part by the joint program of the Office of Naval Research and the Atomic Energy Commission. ** Now at the Institute for Advanced Study; Princeton, New Jersey.

\section{ABSTRACT}

Two Independent methods are described for calculating the multiple scattering distribution for projected angle scattering resulting when very high energy charged particles traverse a thick scatterer. The single scattering law for projected angle scattering is taken to be the Rutherford scattering law for projected angle scattering modified at small angles by electron shielding and at large angles by a nuclear form factor $\mathcal{F}_{n}\left(\varphi / \varphi_{0}\right)$ which gives the effect of the finite nuclear size. The calculations can be carried through for any reasonable choice of of and have been carried through for two suggested choices of $f_{n}$ for the examples of fast $\mu$-meson scattering in $2 \mathrm{~cm}$ and $5 \mathrm{~cm}$ thick lead slabs, with good agreement for the two methods of calculation. The results are compared with the theories of Molière and Olbert. 


\section{SECTION 1. INTRODUCTION}

Several multiple scattering theories have been published $1,2,3,4$

1

E. J. Williams, Proc. Roy. Soc. Al69, 531 (1939);

S. Goudsmit and J. L. Saunderson, Phys. Rev. 58, 36 (I940);

H. S. Snyder and W. T. Scott, Phys. Rev. 76, 220 (1949);

H. W. Lewis, Phys. Rev. 78, 526. (1950).

2 G. Molière, Z. Naturforsh 2a, 133 (1947); 3a, 78 (1948)

3 s. Olbert, Phys. Rev. 87, 319 (1952);

M. Annis, H. S. Bridge, and S. Olbert, Phys. Rev. 89, 1216 (1953)

4 H. A. Bethe, Phys. Rev. 89, 1256 (1953)

which are concerned with the angular distribution of particles passing, with no substantial loss of energy, through a thick material for which the single scattering law is the Rutherford cross section modified at small angles due to electron screening. In this paper we shall present two methods by which a multiple scattering distribution for projected angle scattering can be obtained from more general single scattering laws than those already treated. We shall be concerned in particular with single scattering which is Rutherford for a wide middle region; but is modifled both at large as well as at small angles. In scattering from atoms the modification at small angles is due to electron screening, while at large angles there can be modi-

page two 
fications due to additional forces (as in the scattering of $\pi$ mesons from carbon) or due to the finite extension of the nucleus and the subsequent deviations from Coulomb's law inside the nucleus. We consider only the case where relatively small $\varphi$ are important so the approximation $\varphi \approx \sin \varphi \approx \tan \varphi$ is appropriate.

There has been a good deal of experimental interest recently 5,6

5 E. Amaldi and $G$. Fidecaro, Nuovo Cimento, I, 535 (1.950);

W. L. Whittemore and R. P. Shutt, Phys. Rev. 88, 1312 (1952);

E. P. George, J. L. Reddjing, and P. T. Trent, Proc. Phys. Soc. A66, 533 (1953);

B. Leontic and A. W. Wolfendale, Phil. Mag. 44, 1091 (1953). 6 M. L. T. Kanangara and G. S. Shrikantia, Phil. Mag. 44,1091 (1953).

in the multiple scattering of $\mu$-mesons. Experimental results, after being compared with the results of the Molière ${ }^{2}$ and Olbert ${ }^{3}$ muliple scattering theories have been interpreted as indicating the existence of an anomolous $\mu$-meson-nuclear interaction. Among the many difficulties arising in the interpretation of these experiments (see Appendix B), one of the most obvious seemed to be the absence of a reliable estimate of the expected multiple scattering distribution from extended nuclei.

In the Moliere multiple scattering theory the nucleus is treated as a point charge. The single scattering cross section is taken to be the Rutherford cross section modified, at small 
angles only, due to electron screening. This gives more scattering for large angles than would be expected from extended nuclei. In the olbert theory an attempt is made to estimate the effect of the nuclear extension by multiplying the Molière projected angle single scattering law by a step function which cuts off all scattering beyond a given projected angle $\varphi_{0} \approx X / R$. This, however, gives a very great underestimate of the multiple scattering for angles larger than $\varphi_{0}$, as for large angles Olbert's distribution falls off as a Gausian which soon is much smaller than even the coherent part of the single scattering law.

In what follows we shall outline two distinct procedures by which a multiple scattering distribution can be obtained from given single scattering laws. We deal with projected angle scattering as this is the usual experimental parameter. Our second method could be extended to include the total scattering angle. Sections 2 and 3 describe the two methods we have developed for dealing with multiple scattering problems. The results of these two sections have been used to calculate the expected multiple scattering distribution of relativistic $\mu-m e s o n s(c p=I \mathrm{Bev})$ passing through 2 and $5 \mathrm{~cm}$ of lead. Agreement between the two methods is excellent. $\Lambda$ ppendix A contains a discussion of the single scattering cross section used for the above calculation. Appendix. B gives a review of the experimental situation with regard to $\mu$-meson scattering. 
SECTION 2. DESCRIPTION OF THE FIRST METHOD OF COMPUTING M( $M)$

The first method to be described consists of folding together several simpler distributions in a manner somewhat analogous to the actual effect of successive layers of the scatterer." Although this method is simple in principle, it seemed on first inspection that it would be extremely tedious to carry through such folding operations. This did not prove to be the case when the computing techniques described below were used, and we give a rather detailed description of the procedure for this reason.

The method is based on the observation that if $f(\varphi) d \varphi$, the probability of a single scattering through $\varphi$ to $\varphi+d \varphi$ to one side in projected angle, is given, then the multiple scattering distribution $M(\varphi)$ is completely defined. (Note that $f(\varphi)$ is not normalized to unity, but integration over all angles gives the average number of single scatterings in traversing the sample.) Furthermore, if the actual scattering $\mathrm{slab}$ were replaced by a series of consecutive slabs $A, B, C$ having single scattering laws $f_{A}(\varphi), f_{B}(\varphi), \cdots$, , where

$$
f(\varphi)=f_{A}(\varphi)+f_{B}(\varphi)+\cdots
$$

then the same multiple scattering distribution results on traversing all of the slabs in series. If $M_{A}(\varphi), M_{B}(\varphi)$, - . are the separate multiple scattering distributions for A, B, ...., then $\mathbb{M}(\varphi)$ results on folding $M_{A}, M_{B}, \ldots$ together. 
For the case of two components

$$
M(\varphi)=\int_{-\infty}^{\infty} M_{A}\left(\varphi_{1}\right) M_{B}\left(\varphi-\varphi_{1}\right) d \varphi_{1}
$$

We require that only small $\varphi$ are important so $\sin \varphi \approx \tan \varphi \approx \varphi$, and effectively consider $\varphi$ as ranging from $-\infty$ to $+\infty$.

The distribution law for small angles is just the Rutherford scattering law modified due to electron shielding. Following Mollere we represent this as

$$
f^{\prime}(\varphi)=\frac{Q}{2}\left(\varphi^{2}+\varphi_{m}^{2}\right)^{-3 / 2}
$$

where $\varphi$ is the projected angle and $\mathscr{P}_{m}$ is the screening angle

$$
\varphi_{m}=\frac{1.14 m_{e} c^{2} z^{1 / 3}}{137 \mathrm{cp}}\left[1.13+3.76(z / 137 \beta)^{2}\right]^{1 / 2}
$$

and $Q=4 \pi(N t / A)\left(z \theta^{2} / p v\right)^{2}$. Here $(N t / A)$ gives the scatterer thickness in atoms $/ \mathrm{cm}^{2} ; p$ is the momentum of the incoming particle; $\chi=\hbar / p$; $v$ is the incoming velocity; a is the "FermiThomas radius" of the atom $=1.67 \times 10^{4} \mathrm{z}^{-1 / 3}\left(\theta^{2} / \mathrm{m}_{\theta} \mathrm{c}^{2}\right)$. Z is the atomic number of the scattering material (the incident particle is taken to be singly charged) and $m_{e}$ is the electron mass.

The modification in the above distribution law at larger angles is given by multiplying $f^{\prime}(\varphi)$ above by $\mathcal{F}_{\mathcal{N}}\left(\varphi / \varphi_{0}\right)$ to give

$$
f(\varphi)=\frac{Q}{2}\left(\phi^{2}+\phi_{m}^{2}\right)^{-3 / 2} f_{N}\left(\varphi / \varphi_{0}\right)
$$

$\mathcal{F}_{\mathrm{N}}\left(\varphi / \varphi_{0}\right)$ is discussed in Appendix $A_{0}$. 
The trick of this method consists of selecting some angle $\varphi^{\prime}$ which is a lj.ttle smalier than the R. M. S. width of the gausian which approximates $M(\varphi)$ at small angles. $f_{A}(\varphi)$ is then set equal to $\varphi(\varphi)$ for $|\varphi|\left\langle\varphi^{\prime}\right.$ and $=0$ for $|\varphi| \geqslant \varphi^{\prime}$, while $f_{B}(\varphi)=0$ for $|\varphi|<\varphi^{\prime}$ and $=f(\varphi)$ for $|\varphi| \geqslant \varphi^{\prime}$. The multiple scattering due to $f_{A}$ is given with good accuracy by the gaussian

$$
\begin{aligned}
& M_{A}(\varphi)=\left(2 \pi\left\langle\varphi^{2}\right\rangle\right)^{-1 / 2} e^{-\varphi^{2} / 2\left\langle\varphi^{2}\right\rangle} \\
& \left\langle\varphi^{2}\right\rangle=Q \int_{0}^{\varphi^{\prime}} \varphi^{2}\left(\varphi^{2}+\varphi_{m}^{2}\right)^{-3 / 2} d \varphi
\end{aligned}
$$

where

since $\mathcal{F}_{N}\left(\varphi / \varphi_{0}\right) \approx I$ for $|\varphi| \leqslant \varphi^{\prime}$ : Also $\varphi^{\prime}>>\varphi_{m}$ for a reasonably thick scatterer so we can simplify the resulting expression

$$
\begin{aligned}
\left\langle\varphi^{2}\right\rangle= & Q\left[\frac{-x}{\left(1+x^{2}\right)^{I} / 2}\right]_{0}^{\varphi^{\prime} / \varphi_{m}}+\left[\ln \left(x+\sqrt{1+x^{2}}\right)\right]_{0}^{\varphi^{\prime} / \varphi_{m}} \\
& \left\langle\varphi^{2}\right\rangle \approx Q\left\{\ln \left(2 \varphi^{\prime} / \varphi_{m}\right)-1\right\}
\end{aligned}
$$

It is convenient to use the farameter $y=\varphi / \varphi_{0}$ and replace $f(\varphi), Q, \varphi_{m}$, and $\varphi^{\prime}$ by the equivalent quantities $g(y)$; $\mathrm{B}, \mathrm{y}_{\mathrm{m}}$, and $\mathrm{y}^{\prime}$. For the case of a $\mu$-rieson with $\mathrm{cp}=1$ Bev and a $2 \mathrm{~cm}$ lead scatterer, $\varphi_{0}=0.0304$ radians $=1.74^{\circ}, B=0.126$, and $\mathrm{y}_{\mathrm{m}}=9.6 \times 10^{-4}$. For the choice $\mathrm{y}^{\prime}=0.5$ we obtein $\left\langle\mathrm{y}^{2}\right\rangle=0.75$, showing that this is a satisfactory choice for $y^{\prime}$. Then, for this case,

$$
M_{A I}(y)=(1.50 \pi)^{-1 / 2} e^{-y^{2} / 1.50}
$$


whire the subscript $I$ on $\mathbb{M}_{A I}$ emphasizes that this method pives the distribution in terms of $\mathrm{y}=\phi / \varphi_{0}$. (The distribution iunction $M_{2}(x)$ of the next section is given in terms of the angie characteristic of the Molière theory.)

The multiple scattering distribution $\mathrm{M}_{\mathrm{BI}}(\mathrm{y})$ corresponding to single scattering angles $\geqslant \varphi^{\prime}=y^{\prime} \varphi_{0}$ is obtained using the following considerations. If we chose, not the full scatierer thickness, but some sufficiently small fraction $\alpha$, then the single scattering law $\propto \mathrm{g}(\mathrm{y})$ for $|y|>\mathrm{y}^{\prime}$ would have essentially unit weighting for no scatterings at all, and very smell vreightings for all $|\mathrm{y}|>\mathrm{y}^{\prime}$. The corresponding multiple scattering distribution would then be identical to the single scattering law for $|y|>y^{\prime}$ since there is negliglble likelihood of more than one scattering. If this distribution is folded together with itself the multiple scattering is obtained for fraction $2 \alpha$ of the total thickness. This aistribution differs from the single scattering law by double scattering terms proportional to $\alpha^{2}$, so the fractional deviation is proportional to $\alpha<1$. If the multiple scattering law for thickness $2 \alpha$ is folded together with itself the multiple scattering law for thickness $4 \propto$ results. For $|\nabla|>y^{\prime}$ this distribution has a fractional deviation from double that for $2 \alpha$ of an amount double that of the previous process (always neglecting higher order effects). Thus the net fractional correction from the single scattering law is 3 times that obtained in the first folding. Subsequent foldings for $8 \alpha$, 
$16 \alpha, 32 \alpha$, etc. have net fractional corrections $(1+2+4)$, $(1+2+4+8),(1+2+4+8+16)$, etc. times that of the folding starting with thickness $\alpha$. These sums, 7, 15, 31, ...., would be $8,16,32, \ldots$ if we had started our folding process with an infinitesimal fraction of $\alpha$, since going from $\alpha / 2$ to $\alpha$ gives $1 / 2$, from $\alpha / 4$ to $\alpha / 2$ gives $1 / 4$, etc. for the extra series terms $(1 / 2+1 / 4+\ldots)=$.1 . "We thus note that the net fractional correction after any stage of folding (neglecting higher order effects) is just double the fractional correction obtained by the given step ( $i . \theta$. comparing the multiple scattering for $y \neq 0$ for the given thickness with double that for half thickness). We make use of the above feature to select as our starting thickness a fraction $2^{-n}$ of the total for which the fraction correction of any of the points in the first folding is not too large. This fractional correction is then doubled to account with good accuracy for the effect of not starting the process with an infinitely thin sample. In the calculations for $2 \mathrm{~cm}$ lead and $c p=I$ Bev it was found to be suitable to start with $1 / 8$ the sample thickness, while $1 / 16$ the sample thickness was suitable for $5 \mathrm{~cm}$ lead. The actual folding operations can be carried through with good accuracy by replacing the continuous $f_{B}(\varphi)$ by a discontinuous function having values only at regular grid points. For the $2 \mathrm{~cm}$ lead case where $\mathrm{y}^{\prime}=0.5$, the region 0.5 to 0.7 was represented by $0.2 \mathrm{~g}(0.6)$, etc. so values were defined only at $y_{j}=0, \pm 0.6, \pm 0.8, \pm 1.0, \ldots-$ with a maximum 
$y_{j}=6.0$ used in the calculations. For the $5 \mathrm{~cm}$ lead case we used $y^{\prime}=1.2$ and 0.4 intervals to $y=10.0$. It was found convenient and permissible in these cases to neglect contributions to values of $\mathrm{y} \neq 0$ where $|\mathrm{y}|<\mathrm{y}^{\prime}$. (In cases where these contributions are not negligible it requires only slight additional computing time to include them.) The remaining computing tricks can best be illustrated in terms of the examplo of the calculation for the $2 \mathrm{~cm}$ lead case where we started with $1 / 8$ of the total thickness. Let $\ell_{1 / 8}^{0}\left(y_{j}\right)$ be the lumped weights given to the grid points $0, \pm 0.6, \pm 0.8, \cdots, \pm 6.0$ to represent the single scattering law $(0.2 / 8) \mathrm{g}\left(\mathrm{y}_{j}\right)$ for $\left|\mathrm{y}_{j}\right|>0.5$, with

$$
\ell_{1 / 8}^{0}(0)=1-2 \sum_{y_{j}>0} \quad l_{1 / 8}^{0}\left(y_{j}\right)
$$

A calculation sheet is now prepared in which values of $\mathrm{y}_{j}=$ $0,0.2,0.4, \cdots, 6.0$ are entered in the first column. The values of $\ell_{1 / 8 \mathrm{y}_{j}}^{\circ}$ ) are entered in the second column. An uncorrected multiple scattering distribution $\ell_{1 / 4}^{\circ}(y)$ is then computed where

$$
\ell_{I / 4}^{0}\left(y_{j}\right)=\sum_{k} \ell_{I / 8}^{0}\left(y_{k}\right) l_{I / 8}^{0}\left(y_{j}-y_{k}\right)
$$

Use is made of the fact that terms for $\mathrm{y}_{k} \neq \mathrm{y}_{j} / 2$ appear twice and we can write

$$
l_{I / 4}^{0}\left(y_{j}\right)=2 \sum_{y_{k}<y_{j} / 2} l_{1 / 8}^{0}\left(y_{k}\right) l_{1 / 8}^{0}\left(y_{j}-y_{k}\right)+\left[l_{1 / 8}^{0}\left(y_{j} / 2\right)\right]^{2}
$$

The calculations thus proceed as follows. In the third 
column the values of $\ell_{I / 8}^{0}\left(y_{j}\right)$ are multiplied by $\ell_{\frac{1}{8}}^{0}(0)$. The first number appeaxing in the column ( $y_{j}=0$ here) is circled and is only counted once in the subsequent suming. The next column entry starts at $y_{j}=1.2$ and contains products $\ell_{1 / 8}^{0}(0.6)$ $\ell_{1 / 8}^{0}\left(y_{j}-0.6\right)$. Again the first term at $y_{j}=1.2$ is circled. The next column starts at $\mathrm{y}_{\mathrm{j}}=1.6$ and contains terms $\ell_{1 / 8}^{0}(0.8)$ $\ell_{1 / 8}^{0}\left(y_{j}-0.8\right)$. Subsequent columns are formed similarly to produce a triangular array (requiring less than 1 hour of slide rule computing time). Contributions from negative $y_{k}$ are usually negligible but the terms for the first few negative values of $y_{k}$ can be added if necessary. The rows are then added as indicated by Eq. II to give $l_{1 / 4}^{0}\left(y_{j}\right)$. The function $l_{1 / 4}^{0}\left(y_{j}\right)$ is then compared with $2 \ell_{1 / 8}^{0}\left(y_{j}\right)$ for $y_{j} \neq 0$ and the differences are added to $f_{l / 4}\left(\mathrm{y}_{j}\right)$ to obtain the corrected multiple scattering distribution $l_{1 / 4}\left(y_{j}\right)$. This is similarly folded with itself to give $\ell_{1 / 2}\left(y_{j}\right)$, and a repetition gives $\ell\left(y_{j}\right)$ which corresponds to $M_{B I}(y)$. Values of $M_{A l}(y)(E q .8)$ are computed for $y_{j}=0,0.2$, $0.4,---, 6.0$. The final $\mathrm{M}_{I}(y)$ is just the sum of gaussians centered at $0, \pm 0.6, \pm 0.8, \cdots \cdots \pm 6.0, \cdots$

$$
M_{1}\left(y_{j}\right)=\sum_{y_{k}} l\left(y_{k}\right) M_{A 1}\left(y_{j}-y_{k}\right)
$$

The value of $\mathrm{M}_{2}\left(\mathrm{y}_{j}\right)$ for any $\mathrm{y}_{j}$ is computed using $\mathrm{Eq}$. 12. For $\mathrm{y}_{j}$ near 6.0 the contributions from $\mathrm{y}_{k}>6.0$ must be estimated. Fortunately the single scattering $g(y)$ and $l\left(y_{k}\right)$ are decreasing positive functions of $\mathrm{y}$ so the terms in Eq. 12 have a maximum for $\mathrm{y}_{k}<\mathrm{y}_{\mathrm{j}}$ and the remainder can be estimated by noting the behavior 
of the series for smaller values of $y_{j}$.

The above observations suggest an easy method for estimating $M_{1}(y)$ for large $y$ in terms of $M_{A I}(y)$ and $M_{B I}(y)$ without actually carrying through the process indicated by Eq. I2. If $\mathrm{M}_{\mathrm{BI}}(\mathrm{y})$ can be approximated by a straight line on a semi-log plot of the form

$$
M_{B I}\left(y_{k}+x\right)=M_{B I}\left(y_{k}\right) e^{-x / \theta}
$$

then Eq. 2 gives

$$
M_{I}\left(y_{j}\right)=M_{B I}\left(y_{j}\right) \frac{\left\langle y^{2}\right\rangle}{e^{2 a^{2}}}
$$

This may be regarded as increasing $M_{B I}\left(y_{j}\right)$ by a certain amount on the semi-log plot to produce $M_{l}\left(y_{j}\right)$. Alternatively, it can be written

$$
M_{B I}\left(y_{j}\right)=M_{I}\left(y_{j}+\Delta\right)
$$

corresponding to a sidewise displacement $\triangle$ of $\mathrm{M}_{\mathrm{BI}}\left(\mathrm{y}_{j}\right)$ to generate $\mathrm{M}_{I}\left(\mathrm{y}_{j}+\Delta\right)$. The quantity $\Delta$ is obtained by plotting both $\mathrm{M}_{\mathrm{AI}}(\mathrm{y})$ and $M_{B I}(y)$ on the same semi-log plot as in Fig. 1 . Let $\Delta_{j}^{\prime}$ be the value of $\mathrm{y}$ at which $\mathrm{M}_{\mathrm{Al}}(\mathrm{y})$ has the same (logarithmic) slope that $M_{B I}(\gamma)$ has at $y=y_{j}$. Using Eq. 13 shows that $\Delta^{\prime}=\left\langle y^{2}\right\rangle / a$, so $\Delta=\Delta^{\prime} / 2$. Here a is assumed to be a slowly varying function of $y$. (The plot by comparison can be made rapidly, using two straight edges.) When the semi-log plot of $\mathrm{M}_{\mathrm{BI}}(\mathrm{y})$ also has curvature so

$$
M_{B I}\left(y_{k}+x\right) \approx M_{B I}\left(y_{k}\right) e^{-x / a} e^{x^{2} / 2 b^{2}}
$$


then the equation analogous to $\mathrm{Eq} .14$ is

$$
M_{I}\left(y_{j}\right) \approx M_{B I}\left(y_{j}\right)\left[b^{2} /\left(b^{2}-\left\langle y^{2}\right\rangle\right)\right]^{1 / 2} \cdot \frac{b^{2}\left\langle y^{2}\right\rangle}{2 a^{2}\left(b^{2}-\left\langle y^{2}\right\rangle\right)}
$$

which, for $\left.\left.b^{2}\right\rangle\right\rangle\left\langle\mathrm{y}^{2}\right\rangle$ can be written approximately as

$$
M_{I}\left(y_{j}\right) \approx M_{B I}\left(y_{j}\right) e^{\frac{\left\langle y^{2}\right\rangle}{2 z^{2}}\left[I+\left(a^{2}+\left\langle y^{2}\right\rangle\right) / b^{2}\right]}
$$

Inspection of the final result shows that this corresponds again to a sidewise displacement of $M_{B I}$ by $\Delta \approx 0.6 \Delta^{\prime}$ to generate $M_{1}$ in the examples considered. An alternate method of sidewise displacement is obtained by noting that $M_{A I}(x) M_{B I}(y-x)$ has its maximum at $\Delta^{\prime}$, about which it resembles a displaced gaussian. This suggests that $M_{I}\left(y_{j}\right)$ is generated mainly from $M_{B I}\left(y_{k}\right)$ in the region $\mathrm{y}_{k} \approx \mathrm{y}_{j}-\Delta^{\prime}$ so $M_{I}(y)$ should be generated using $M_{B I}\left(y-\Delta^{\prime}\right)$ multiplied by $e^{-\Delta^{\prime 2} / 2\left\langle y^{2}\right\rangle}$. (The last factor is the ratio $M_{A I}\left(\Delta^{\prime}\right) /$ $\left.\mathrm{M}_{\mathrm{AI}}(0)_{0}\right)$ These methods of generating the approximate curve for $\mathrm{M}_{I}(y)$ can be carried out rapidly by simple displacements on the semi-log plot and are quite instructive in giving insight into the behavior of $M_{I}(y)$. The difference between $\mathbb{M}_{B I}(y)$ and $g_{B}(y)$ decreases rapidly if the choice of the dividing angle $y^{\prime}$ is increased. This is compensated largely by an increase in the width of the gaussian $\mathrm{M}_{\mathrm{Al}}(\mathrm{y})$, and thus in the required sidewise displacement $\Delta^{\prime}$ to generate $M_{I}(\eta)$ from $M_{B I}(y)$. It is of interest that the second method (of the next section), at large angles, just folds the single scattering law at large angles with a 
gaussian characteristic of the Molière theory, which is somewhat wider than $M_{A I}(y)$ for the method of selecting $y^{\prime}$ discussed above. Figs. 1, 2, and 4 show the characteristic functions when only elastic processes are included. It is seen that $\mathrm{M}_{1}(\mathrm{y}) \mathrm{re-}$ mains considerably greater than $\delta_{B}$ in a region where the Moliere curve is almost the same as the point nucleus single scattering curve. This is consistent with the above discussion since the logarithmic slope of the point nucleus $g(y)$ is much smaller than the extended nucleus $g(y)$ so the required sidewise displacement Is less, and the result of a given sidewise displacement is also less (using Eq. 15.). When inelastic scattering is included, Figs. 3 and 5, the curves approach those for a point nucleus, decreased by a factor $\mathrm{z}^{-1}$ at very large $\mathrm{y}$.

It is instructive to make a further comparison of this method and the second method (of the next section) for $\mathrm{y}>4$, say. This method folds together the small angle multiple scattering gausian with the large angle multiple scattering distribution, while the second method essentially folds together a wider gaussian and the law for single scattering. The second method uses an expansion in terms of the parameter $(4 G)^{-1}$ described below, neglecting terms of order $(4 G)^{-2}$ and beyond. This very roughly corresponds to neglecting multiple large angle scattering, so the first method would be expected to be more reliable in cases where such effects are not negligible.

As a final point of interest, we note that the curves given in terms of $\mathrm{y}$ apply for all relativistic momenta where $\beta \approx I$. 
In the example considered; $y$ is in units of 1.74 Bev degrees. Thus multiplying the absissa, and dividing the ordinate by 1.74 gives the distribution per Ber degree unit of ( $c p \varphi$ ). This is shown by noting that $f(\varphi) d \varphi=g(y) d y$ so

$$
\begin{aligned}
& g(y)=\frac{B}{2}\left(y^{2}+y_{m}^{2}\right)-3 / 2 \gamma_{N}(y) \text { where } \\
& B=Q \varphi_{0}^{-2}=4 \pi\left(\frac{Z R}{137 \beta}\right)^{2}\left(\frac{N t}{A}\right) \\
& y_{m}=P_{m} / \varphi_{o}=\frac{1.14}{137^{2} r_{o \theta}}\left[1.13+3.76(\mathrm{z} / 137 \beta)^{2}\right]^{1 / 2} \text { and } \\
& r_{0 \theta}=\theta^{2} / m_{\theta} c^{2} \approx 2.82 \times 10^{-13} \mathrm{~cm} \text { are Independent of } \mathrm{p} \text { for }
\end{aligned}
$$

$\beta \approx I$

\section{SECTION 3. MODIFIED MOLIERE THEORY}

\section{A. Methods and Notation}

To introduce the mathematical methods and notation used in this section we review the derivation of the general expression for the projected angle multiple scattering distribution for an arbitrary single scattering cross section. The derivation follows that of Moliere ${ }^{2}$ and 0 lbert $^{3}$ and applies whon only relatively small angles are important:

If $f(\varphi) d \varphi$ is the probability that an incident particle undergo a single scattering through the profected angle $\varphi$ to $\varphi+d \varphi$ 
$R-83$

In Its passage through the scatterer, then

$$
P\left(\varphi_{1}, \varphi_{2}, \cdots \varphi_{n}\right) d \varphi_{1} \cdots d \varphi_{n}=e^{-\sigma_{T}} f\left(\varphi_{1}\right) f\left(\varphi_{2}\right) \cdots f\left(\varphi_{n}\right) d \varphi_{1} d \varphi_{2} \cdots d \varphi_{n}
$$

where $\sigma_{t}=\int_{-\infty}^{\infty} f(\varphi) d \varphi$, is the probability that the particle in passing through the material has exactly $n$ single scatterings through the projected angles $\varphi_{1}, \varphi_{2}, \ldots-, \varphi_{n}$ with angular intervols $d \varphi_{1}, d \varphi_{2}, \ldots-\cdots, d \varphi_{n} \cdot e^{-\sigma_{t}}$ is the probability that there be no other scatterings besides $\varphi_{1}, \varphi_{2}-\infty \varphi_{n}$. Since we are interested only in the final angle $\varphi$ we integrate over intermediate angles and put

$$
P^{(n)}(\varphi) d \varphi=\frac{d \varphi}{n !} e^{-\sigma_{T}} \int_{-\infty}^{\infty} \cdots \int f\left(\varphi_{1}\right) f\left(\varphi_{2}\right) \cdots f\left(\varphi-\varphi_{1}-\varphi_{2} \cdots-\varphi_{n-1}\right) d \varphi_{1} \cdots d \varphi_{n-1}
$$

where $P^{(n)}(\varphi) d \varphi$ is the probability that the particle emerge at a projected angle between $\varphi$ and $\varphi+d \varphi$ after exactly n scatterings in the material. The factor $I / \mathrm{n}$ l occurs because in the integration over intermediate angles the permutations of $\varphi_{1}$

$\varphi_{n}$ have each been counted. Since Eq. 18 holds for independent events the order of the $n$ scatterings is irrelevant.

Now introduce the Fourier representation of the single scattaring cross section. Let

$$
\begin{aligned}
& f(\varphi)=\frac{1}{2 \pi} \int_{-\infty}^{\infty} g(\xi) e^{i \xi \varphi} d \xi \\
& g(\xi)=\int_{-\infty}^{\infty} f(\varphi) e^{-i \xi \varphi} d \varphi
\end{aligned}
$$

so

(21)

page sixteen

$3: 28$ 
(where $g(\xi)$ Is not related to $g(y)$ of the provious section). Putting Eq. 20 into Eq. 19 gives

$P^{(n)}(\varphi) d \varphi=\frac{d \varphi}{n !} e^{-\sigma_{T}}(2 \pi)^{-n} \int_{-\infty}^{\infty} \cdot \int_{-\infty} g\left(\xi_{1}\right) \cdots g\left(\xi_{n}\right) e^{i \xi_{1} \varphi_{1}} \cdots e^{i \xi_{n}\left(\varphi-\varphi_{1} \cdots-\varphi_{n-1}\right)} d \xi_{1} \cdots d \xi_{n} d \phi_{1} \cdots d \varphi_{n-1}$

The Integrations over $\varphi_{1}, \ldots \varphi_{n-1}$ give delta functions which, after the $\xi_{I} \ldots \xi_{n-1}$ integrations yield

$$
P^{(n)}(\varphi) d \varphi=\frac{d \varphi}{2 \pi} e^{-\sigma_{T}} \int_{-\infty}^{\infty} \frac{[g(\xi)]^{n}}{n !} e^{i \xi \varphi} d \xi
$$

The probability of a final projected angle $\varphi$ after any number of scatterings is just the sum of the $P^{(n)}(\varphi) d \varphi$ over all values of $n$.

$P(\varphi) d \varphi \equiv \sum_{n=0}^{\infty} P(\varphi) d \varphi=\frac{d \varphi}{2 \pi} e^{-\sigma_{T}} \int_{-\infty}^{\infty} \sum_{n=0}^{\infty} \frac{[g(\xi)]^{n}}{n !} e^{i \xi \varphi} d \xi$

where we use the result from $\mathrm{Eq} .21$

$$
\sigma_{t}=\int_{-\infty}^{\infty} f(\varphi) d \varphi=g(0)
$$

From this it can be seen that the solution of the multiple scattering problem is equivalent to the evaluation of the integral In Eq. 24. Mollère ${ }^{2}$ has given an evaluation of this integral In the case of a screened Coulomb field. The main trick in evaluating the integral lies in the observation that the existence of multiple scattering will smear out fine grained irregularities In the final distribution so the high Fourler components give a negligible contribution.

Mollere used the single scattering law of Eq. 3 appropriate 
for a point nucleus with electron shielding. We are interested in the case where this is modifled by the nuclear "form factor" $\mathcal{F}_{N}\left(\varphi / \varphi_{0}\right)$ as in Eq. 4. From the discussion of Appendix $A$ we note that

$$
\mathcal{F}_{N}\left(\varphi / \varphi_{0}\right) \approx\left\{\begin{array}{l}
1 \text { for small }\left|\varphi / \varphi_{0}\right| \\
z^{-1} \text { for large }\left|\varphi / \varphi_{0}\right|
\end{array}\right. \text { (our choice) }
$$

with the rapid change occuring when $\varphi \sim 2 \varphi_{0}$. olbert's method consisted of using a step function for $\mathcal{F}_{N}$ $\mathcal{f}_{N}\left(\varphi / \varphi_{0}\right)= \begin{cases}1 & \text { for }|\varphi| \leqslant \varphi_{0} \\ 0 & \text { for }|\varphi|>\varphi_{0}\end{cases}$

As mentioned in the introduction, this gives a very large underestimate of the multiple scattering for $\varphi>>\varphi_{0}$ where the olbert function falls off as a gaussian while, for any reasonable form factor; the multiple scattering distribution lies above the single scattering distribution $f(\varphi)$ for large $\varphi$, and thus far above the Olbert distribution.

\section{B. First Derivation of $\mathrm{M}_{2}(\mathrm{x})$}

We first treat the multiple scattering from extended nuclei as a correction to the Molière theory by setting $\mathcal{F}_{N}=\left[1+\left(\mathcal{F}_{N}-1\right)\right]$; where $\left(\mathscr{F}_{N}-1\right)$ gives the correction term. This is the method used by Olbert for his step function. Such a treatment yields satisfactory results for small angles, but is inconvenient for 
large angles where a second method (described following this) is preferred. Eq. 4 1s written

$f(\varphi)=\frac{Q}{2}\left(\varphi^{2}+\varphi_{m}^{2}\right)^{-3 / 2}\left[1+\left(\mathcal{F}_{N}-1\right)\right]$

$g(\xi)-g(0)=\frac{Q}{2} \int_{-\infty}^{\infty} \frac{\left(e^{-i \xi \varphi}-1\right) d \varphi}{\left(\varphi^{2}+\varphi_{m}^{2}\right)^{3 / 2}}+\frac{Q}{2} \int_{-\infty}^{\infty} \frac{\left(e^{-i \xi \varphi}-1\right)\left(\mathcal{F}_{N}-1\right) d \varphi}{\left(\varphi^{2}+\varphi_{m}^{2}\right)^{3 / 2}}$

The first integral has been treated by Mollère ${ }^{7}$ and to a

7

See H. A. Bethe, Reference 4, for a discussion of this integral.

sufficient degree of accuracy, is

$\frac{Q}{2} \int_{-\infty}^{\infty} \frac{\left(e^{-i \xi \varphi}-1\right) d \phi}{\left(\varphi^{2}+\varphi_{m}^{2}\right)^{3 / 2}} \approx Q\left[\frac{\xi^{2}}{4} \ln \frac{\gamma^{2} \varphi_{m}^{2}}{4 e} \xi^{2}\right]$

We now Introduce the various parameters typical of the Molière theory, following the notation of 0lbert.

$G=-\frac{1}{2} \ln \left(\frac{\gamma^{2} \varphi_{m}^{2}}{e} \frac{1}{2 G \varphi}\right) ; x=(2 G Q)^{-1 / 2} \varphi ; \eta=(2 G Q)^{1 / 2} \xi$

and $G \approx 5.66+1.24 \log _{10}\left[\frac{Z^{4 / 3} A^{-1} t}{1.13 \beta^{2}+3.76(Z / 137)^{2}}\right]$

is independent of the momentum in the relativistic region.

Putting Eq. 29 in 28 , and using 30 gives

$s(\eta) \equiv g\left(\frac{\eta}{\sqrt{2 G Q}}\right)-g(0)=-\frac{\eta^{2}}{4}+\frac{1}{2 G}\left[\frac{\eta^{2}}{4} \ln \frac{\eta^{2}}{4}+\frac{1}{2} I\right]$

where $I=\int_{-\infty}^{\infty} \frac{\left(e^{-i \eta x^{\prime}}-1\right)\left[\mathcal{F}_{N}\left(x^{\prime} / x_{0}\right)-1\right] d x^{\prime}}{\left(x^{\prime 2}+x_{m}^{2}\right)^{3 / 2}}$ 
Inserting this result into $\mathrm{Eq}$. 24 we obtain in terms of the $M_{2}(x) d x=\frac{d x}{2 \pi} \int_{-\infty}^{\infty} d \eta e^{i \eta x} e^{-\eta^{2} / 4+\frac{1}{2 G}\left[\frac{\eta^{2}}{4}+\frac{1}{2} I\right]}$

We use the fact that $G$ is a reasonably large number (for moderate foil thickness $G$ varies from about 7 to 15 ) and expand the portion of the exponential which is multiplied by $(2 G)^{-1}$. This gives, to first order in $(2 G)^{-1}$,

$M_{2}(x) d x=\frac{d x}{2 \pi} \int_{-\infty}^{\infty} d \eta e^{i \eta x-\eta^{2} / 4}\left[1+\frac{1}{2 G}\left(\frac{\eta^{2}}{4} \ln \frac{\eta^{2}}{4}\right)+\frac{1}{4 G} I+\cdots\right]$

After performing the integrations over $\eta$ this becomes

$$
M_{2}(x) d x=\frac{e^{-x^{2}} d x}{\sqrt{\pi}}+\frac{d x}{4 G}\left[f^{\prime}(x ; \infty)-K(x)\right]
$$

$K(x)=\frac{1}{\sqrt{1 T}} \int_{0}^{\infty} \frac{d x^{\prime}\left[1-\mathcal{F}_{N}\left(x^{\prime} / x_{0}\right)\right]}{\left(x^{\prime 2}+x_{m}^{2}\right)^{3 / 2}}\left\{e^{-\left(x+x^{\prime}\right)^{2}}+e^{-\left(x-x^{\prime}\right)^{2}}-2 e^{-x^{2}}\right\}$

and

$$
f^{\prime}(x ; \infty)=\frac{1}{\pi} \int_{-\infty}^{\infty} d \eta e^{i \eta x-\eta^{2} / 4}\left(\frac{\eta^{2}}{4} \ln \frac{\eta^{2}}{4}\right)
$$

$f^{\prime}(x ; \infty)$ is the Moliere function. The integral $K(x)$ which oc curs in Eq. 34 must be evaluated numerically for a given $\mathcal{F}_{N}\left(x^{\prime} / x_{0}\right)$. Eq. 34 is seen to have the form that was desired originally. The integral which contalns the effect of the nuclear extension occurs as a correction to $\theta^{-x^{2}} / \sqrt{\pi}+\frac{1}{4 G} f^{\prime}(x ; \infty)$ which is the Molière multiple scattering distribution for a point nucleus in 
terms of the projected angle parameter $x$. This corresponds to $\mathcal{F}_{N}=1$ for all $x$. If $\mathcal{F}_{N}\left(x / x_{0}\right)$ is the 0lbert stop function, Eq.26, the correction term becomes the olbert correction function $K\left(x ; x_{0}\right)$ If use is made of the fact that $x_{0}>>x_{m}$.

Using Eq: 34 the multiple scattering distribution can be determined for a particular $\mathcal{F}_{N}\left(\varphi / \varphi_{0}\right)$ : However, Eq. 34 becomes inconvenient for large values of $x$, because in that case $K(x)$ becomes almost equal to $f^{\prime}(x ; \infty)$ and the difference between two large numbers must be used to give a small one. For large values of $x$ therefore it is better to treat the modified cross section directly, rather than as a correction to the Molière distribution. This is done as below.

\section{SECOND DERIVATION OF $\mathrm{M}_{2}(\mathbf{X})$ !}

Consider again the Fourier transform of the single scattering

$g(\xi)-g(0)=\frac{Q}{2} \int_{-\infty}^{\infty} \frac{\left(e^{-i \xi \varphi^{\prime}}-1\right) \mathcal{F}_{N}\left(\varphi^{\prime} / \varphi_{0}\right) d \varphi^{\prime}}{\left(\varphi^{\prime 2}+\varphi_{m}^{2}\right)^{3 / 2}}$

Introducing the Moliere parameters $x, \eta$ and $G$ as defined Eq. 30 we get

$g\left(\frac{\eta}{\sqrt{2 G Q}}\right)-g(0) \equiv s(\eta)=\frac{1}{2 G} \int_{0}^{\infty} \frac{\frac{\partial}{N}\left(x^{\prime} / x_{0}\right)\left(\cos \eta x^{\prime}-1\right) d x^{\prime}}{\left(x^{\prime 2}+x_{m}^{2}\right)^{3 / 2}}$

and

$$
M_{2}(x) d x=\frac{d x}{2 \pi} \int_{-\infty}^{\infty} e^{i \eta x} e^{\mu(n)} d \eta
$$

page twenty-one 
Eq. 36 of course is the Moliere-Fourier transform if of $\left(x^{\prime} / x_{0}\right)=1$. In the case of the point nucleus this integral is evaluated through the observation that there exists an angle $x^{\prime}=K$ at which the integral can be split such that $k \gg x_{m}$ but $k \ll \frac{1}{\eta_{c}}$ where $\eta_{c}$ is the frequency in the neighborhood of the main Fourler components.4 Then in the integration up to $H$ the frctor $\left(\cos \eta x^{\prime}-1\right)$ can be put equal to $-\left(\eta x^{1}\right)^{2} / 2$ and, in the integration from $K$ to $\infty ;\left(x^{2}+x_{m}^{2}\right)^{-3 / 2}$ becomes $x^{-3}$. Both integrations can be performed, and the splitting point $H$ cancels up to terms of the order $K^{2}$; whlch are very small.

In the case with which we deal the argument is modified as follows. Again we split the integral at an angle $K$ where $x_{m} \ll$ $x^{\prime}=k \ll \eta_{c}^{-1}$. Now we make use of the important property of the form factor given in Eq. 25 that $\mathcal{F}_{N}\left(x_{1} / x_{0}\right) \approx 1$ for small values of the argument $x^{\prime} / x_{0}$. In particular, for $x^{\prime}=1 / 4$ our chosen form of $\mathcal{F}_{N}$ (Appendix A) gives $\mathcal{F}_{N}(1 / 4) \approx 0.96$ for the $2 \mathrm{~cm}$ lead case, and 0.93 for the $5 \mathrm{~cm}$ lead case. The integral up to $K$ is thus the same as in the case of the point nucleus. $s(\eta)=-\frac{1}{2 \cdot 5} \int_{0}^{K} \frac{\left(\eta x^{\prime}\right)^{2} d x^{\prime}}{2\left(x^{\prime 2}+x_{m}^{2}\right)^{3 / 2}}+\frac{1}{2 G} \int_{K}^{\infty} \frac{\mathcal{J}_{N}\left(x^{\prime} / x_{0}\right)\left(\cos \eta x^{\prime}-1\right) d x^{\prime}}{x^{\prime 3}}$

Performing the first integration and using the fact that $x_{m} \ll K$; Eq. 37 becomes

$$
s(\eta)=-\frac{\eta^{2}}{4}-\frac{\eta^{2}}{4 G} \ln (k / 1,26)+\frac{1}{2 G} \int_{K}^{\infty} \frac{\mathcal{J}_{N}\left(x^{\prime} / x_{0}\right)\left(\cos \eta x^{\prime}-1\right) d x^{\prime}}{x^{\prime 3}}
$$

where $\ln (1 / 1.26)=(\ln 2 \gamma-3 / 2)$ 
It is understood that $k$ must bo much smaller than 1. If now $s(\eta)$ is put into Eq. $36 \mathrm{a}$ and the terms multiplied by $(2 \mathrm{G})^{-1}$ are expanded in the same way as before $\theta_{\lambda}$ the $\eta$ integrations, the following result is obtained for the first order in $(2 G)^{-1}$.

$$
M_{2}(x)=\frac{e^{-x^{2}}}{\sqrt{\pi}}\left[1+\frac{1}{4 G} 2\left(2 x^{2}-1\right) \ln (H / 1.26)\right]+\frac{1}{4 G} \frac{1}{\sqrt{\pi}} N(K, x)
$$

where $\quad N(K, x)=\int_{K}^{\infty} \frac{\mathcal{F}_{N}\left(\lambda \mid x_{0}\right)}{\lambda^{3}} T(x, \lambda) d \lambda$

$$
T(x, \lambda)=e^{-(x+\lambda)^{2} \lambda^{3}}+e^{-(x-\lambda)^{2}}-2 e^{-x^{2}}
$$

In 1ts present form Eq. 39 is conventent for calculation only for large values of $x(x \geq 4$ in the $5 \mathrm{~cm}$ lead case) because in this case $e^{-x^{2}}$ is so small that the precise value of $K$ does not influence the result.

However, for smaller values of $x$ the exact value of $K$ is of Importance. In order that the expansion of $\left(\cos \eta x^{i}-1\right)$ in Eq. 37 be correct $K$ mist be much smaller than 1: However, it is inconvenient to evaluate the final integral of Eq. 39 numerically for very small values of $K^{8}$

8 We can see more clearly what error is introduced if $K$ is allowed to become large by doing the following. Expand $T(x, \lambda)$ of Eq. 39 in powers of $2 x \lambda$. Then neglecting terms of order $\lambda^{4}$ or $(x \lambda)^{4}$ gives $\mathrm{T}(\mathrm{x} ; \lambda) \approx 2 \lambda^{2} e^{-x^{2}}\left(2 \mathrm{x}^{2}-1\right)$ 
Integrating $N(K, x)$ from $K$ to $L$ and letting $\operatorname{\alpha f}_{N}\left(\lambda / x_{0}\right)=1$ in this integral we obtain

$M_{2}(x)=\frac{1}{\sqrt{\pi}}\left[e^{-x^{2}}+\frac{1}{4 G} 2 e^{-x^{2}}\left(2 x^{2}-1\right) \ln (L / 1.26)+\frac{1}{4 G} N(L, X)\right]$

But this is just the same result obtained by letting $K=L$ in the Fourier transform $s(\eta)$ of Eq. 37. Thus allowing $K$ to become large introduces the same error as would be introduced if the final result were expanded in powers of $2 \times \lambda$.

To overcome this difficulty one can use the property of the form factor that $\mathscr{F}_{N}\left(\lambda / x_{0}\right) \approx I$ for values of $\lambda$ much larger than $\eta_{c}^{-1}$. Then $\int_{1 / 4}^{L} \lambda^{-3} T(x, \lambda) d \lambda$ can be evaluated analyticaily where $I \approx{ }^{1 / 4}{ }_{T_{h i s}}$ makes the numerical integration $\int_{L}^{\infty} \lambda^{-3} T(x, \lambda) \mathscr{F}_{N}\left(\lambda / x_{0}\right) d \lambda$ practicable. In doing this it will be seen that the dependence upon $K$ vanishes and a conventent and accurate expression for $M_{2}(x)$ 1s obtained. We observe that if $L \approx 1 / 4$ then $\lambda$ can be considered small even if $2 x \lambda$ is not. Thus we can expand $e^{-\lambda^{2}}$ $\ln T(x, \lambda)$.

$T(x, \lambda)=2 e^{-x^{2}} e^{-\lambda^{2}}(\cosh 2 \lambda x-1) \approx 2 e^{-x^{2}}\left(1-\lambda^{2}+\cdots\right)(\cosh 2 \lambda x-1)$ taking only terms up to $\lambda^{2}$. We evaluate $J=\int_{K}^{L} \lambda^{-3} T(x, \lambda) d \lambda \approx 2 e^{-x^{2}} \int_{K}^{L}\left[\frac{(\cosh 2 \lambda x-1)}{\lambda^{3}}-\frac{\cosh 2 \lambda x}{\lambda}\right] d \lambda$ This, after some labor, neglecting terms in $K^{2}$ or higher, gives 
$\begin{aligned} J & \approx e^{-x^{2}}\left[\frac{1}{L^{2}}-\frac{\cosh 2 L x}{L^{2}}-\frac{2 x}{L} \sinh 2 L x\right]+6 x^{2} e^{-x^{2}}+ \\ & +2 e^{-x^{2}}\left(2 x^{2}-1\right)\left[\int_{0}^{2 L x} \frac{(\cosh t-1)}{t} d t+\ln L\right]-2 e^{-x^{2}}\left(2 x^{2}-1\right) \ln k\end{aligned}$

Putting this result into Eq. 39 gives

$M_{2}(x)=\frac{e^{-x^{2}}}{\sqrt{\pi}}\left[1+\frac{g(L, x)}{4 G}\right]+\frac{1}{4 \sigma} \frac{1}{\sqrt{\pi}} \int_{L}^{\infty} \lambda^{-3} \mathcal{F}_{N}\left(\lambda / x_{0}\right) T(x, \lambda) d \lambda(43)$ where 9

$$
\begin{aligned}
g(L, x) & =2\left(2 x^{2}-1\right)\left[\ln (L / 1.26)+\int_{0}^{2 L x} \frac{(\cosh t-1)}{t}+6 x^{2}+\right. \\
& -\frac{1}{L^{2}}(\cosh 2 L x-1)-\frac{2 x}{L} \sinh 2 x L
\end{aligned}
$$$$
\int_{0}^{2 L x} \frac{(\cosh t-1)}{t} \approx \frac{(2 L x)^{2}}{2.2}+\frac{(2 L x)^{4}}{4 \cdot 4 !}+\cdots
$$

and

9 In our earlier communication by the same title, Leon N. Cooper and Jemes Rainwater, Phys. Rev. 95, 1107 (1954), $q(L, x)$ was given as $2\left(2 x^{2}-1\right) q(K)=2\left(2 x^{2}-1\right) \ln (K / 1.26)$ which was a sufficiently good approximation in the example there considered.

\section{EVALUATION OF THE INTEGRALS}

The integrals $K(x)$ and $N(L, x)$ which occur in expressions for the multiple scattering distributions derived above can easily be evaluated by numerical means. $N(L, x)$ in particular can be evaluated conveniently for values of $L=1 / 4$ or $1 / 2$, and for such 
values of $L E q .43$ is accurate. These integrals do not depend on the momentum of the incoming particle but rather on $\beta$, which $\approx I$ in the relativistic region. Thus a single computation is sufficient for all relativistic momenta. For different thicknesses, or atomic number, the form factor is changed only through the change in $x_{0}$ in $\mathcal{F}_{N}\left(x / x_{0}\right)$. Table 1 gives the function

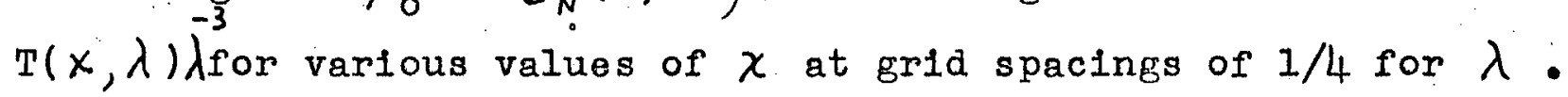
Table 2 gives the values of the function $q(L, X)$ for $L=1 / 4$ for relevant values of $X$ :

$K(x)$ and $N(L, X)$ have been evaluated numerically for the $2 \mathrm{~cm}$ and $5 \mathrm{~cm}$ lead cases for grid spacings of $\Delta x=1 / 2$ and $1 / 4$ using Weddle's rule:10 Comparison of the results for the two

10 H. Margenau and G. M. Murphy, "The Mathematics of Physics and Chemistry", D. Van Nostrand Company, Inc., New York, N. Y., 1943, page 461 .

grids shows the largest numerical errors occur for the small values of $X$ where the correction term is unimportant. For larger values of $x(x \geqslant 3)$ the change in the numerical results for grid changes from $1 / 2$ to $1 / 4$ is less than 2 percent. In all, the errors in the distribution due to numerical errors resulting from the Integration, for a grid of 1/4, appear to be less than 1 percent.

For very large values of $X$ asymptotic formulas can be developed. For a form factor which decreases asymptotically as 
const. $/ x^{n-3}$ the asymptotic expression is

$$
\sigma_{M}=\sigma_{S}\left[1+\frac{n(n+1)}{4 x^{2}}+\cdots--\right]
$$

Such expressions give reasonably accurate results for $x \geqslant 6$ (5 percent or better): The procedure described near the end of section 2 is also readily adapted to this method for a quick graphical correction procedure to obtain $\mathrm{M}_{2}(\pi)$ from the single scattering law. For real atoms at the larger angles incoherent scattering predominates so that the form factor becomes $\mathscr{F}_{N}\left(\varphi / \varphi_{0}\right) \approx \mathrm{z}^{-1}$. Then the multiple scattering distribution is given by the asymptotic expansion of the Moliére distribution multiplied by $z^{-1}$. This is

$$
M_{2}(x) d x=\frac{\sigma_{3}}{z}\left[1+\frac{3}{x^{2}}+\frac{45}{x^{4}}+\cdots--\right]
$$

The results presented in this section have included only the first powers in $(2 G)^{-1}$. It is possible to obtain the next order, but in view of the large uncertainty in the nuclear form factor $\mathcal{F}_{N}\left(\varphi / \varphi_{0}\right)$ it was not considered worthwhile at present to consider this term.

We wish to thank Professor Robert Serber for helpful disthe

cussions of $\wedge$ single proton form factor. We also. wish to thank Miss Hilda Oberthal for her assistance with the computations of the results of the modified Molière theory. 


\section{APPENDIX A。 THE SINGLE SCATTERING LAW}

The single scattering laws used in the calculations were obtained in the following way. The Rutherford formula, modifled at small angles due to eloctron shielding, is given in $\mathrm{Eq} .3$ following Molière. When nuclear extension is considered, this must be multiplied by a nuclear form factor $\mathcal{F}_{N}\left(\varphi / \varphi_{0}\right)$ which contains a part representing elastic coherent scattering plus a part representing inelastic scattering $\mathcal{F}_{N}\left(\varphi / \varphi_{0}\right)=F_{N}^{c}\left(\varphi / \varphi_{0}\right)+F_{N}^{I}\left(\varphi / \varphi_{0}\right)$ In principle, if the nuclear wave functions were known exactly, one would prefer to calculate $F_{N}^{C}$ by on exact phase shift analysis for spin $1 / 2$ particles, and calculate $F_{N}^{I}$ by considering in detail all of the possible final states of the scattering system. However, the nuclear charge distribution, and the nuclear wave functions are not known exactly, and, in fact, measurements of $\mathrm{F}_{\mathrm{N}}^{\mathrm{C}}$ for fast electrons ${ }^{11}$ are providing valuable information con-

Il R. W. PIdd, C. I. Hammer, and E. C.Raka, Phys. Rov. 92 , 436 (1953);

R. Hofstadter, H. R。 Fechter, and J. A. McIntyre, Phys. Rev. 92, 978 (1953);

I. I. Shiff, Phys。Rovo 92, 988 (1953);

R. Hof stadter, B. Hahn, A. W. Knudsen, and J. A. McIntyre, Phys。Revo 95, 512 (1954);

D. R。 Yennie, D. G. Ravenhall, and R.N. Wilson, Phys, Rev。 95 , $500(1954)$ 
cerning the nuclear charge distribution. In particular, mumesonic $x$-ray studies, ${ }^{12}$ fast electron scattering ${ }^{11}$ and other

12

Val. L. Fitch and James Rainwater, Phys. Rev. 92, 789 (1953);

I. N. Cooper and E. M. Henley, Phys. Rev. 92, 801 (1953);

John A. Wheeler, Phys. Rev. 92, 812 (1953);

D. I. H1ll and K. W. Ford, Phys. Rev. 94, 1617 and 1630 (1954)

experimental results ${ }^{12}, 13$ which are sensitive to the nuclear

13

F. Bitter and H. Feshbach, Phys. Rev. 92, 837 (1953);

B. G. Jancovici, Phys. Rev. 95, 389 (1954);

D. C. Peaslee, Phys. Rev. 95, 717 (1954)

charge distribution, all agree that the nuclear charge distribution is more compact than had previously been believed to be the case. For a nuclear charge distribution $\rho(r)=\rho_{0}$ for $r<R=r_{0} A^{l / 3}$, and $\rho(r)=0$ for $r>R$ the radius $R$ has clear meaning and a best match to the above experiments gives $r_{0} \approx 1.0$ to $1.2 \times 10^{-13} \mathrm{~cm}$ for not too small A. Elementary considerations of quantum mechanics show that such a model cannot be strictly correct, and $\rho(r)$ must be a continuous function of $r$. In this case, although the different experiments are not always sensitive in the same manner to the shape of $\rho(r)$, the eguivalent unfform model $r_{0}$ is usually taken to be that value which gives the same $\left\langle\mathrm{r}^{2}\right\rangle$ as for the non-uniform $\rho(r)$. Prior to late 1.953 the 
value of $r_{0}$ would have boen consldered to be "well known" and of magnitude 1.4 to $1.5 \times 10^{-13} \mathrm{~cm}$. Thus all of the comparisons 5.6 between the experimental and expected multiple scattering distributions used this larger value of $r_{0}$.

Until recently it has been customary to calculate $F_{N}^{C}$ using the Born approximation which gives a linear superposition of the scattering amplitudes of the individual protons so the scattered intensity is of the form

$$
A^{2}\left|\left\langle i\left|\sum_{j} e^{-i \vec{q} \cdot \vec{r}_{j}}\right| i\right\rangle\right|^{2}=Z^{2} A^{2} F_{N}^{c}
$$

where $A^{2}$ is the scattering intensity of a single proton, $\vec{q}=\vec{k}-\vec{k}_{0}$ represents the vector momentum change in scattering, $\vec{r}_{j}$ is the position coordinate of the $f^{\text {th }}$ proton in the nucleus, and the ovaluation is for the ground state $|i\rangle$ of the nucleus. Thus $\left(F_{N}^{C}\right)^{1 / 2}$ is just the Fourler transform of the nuclear charge distribution. For a unfform nuclear model, letting $\nabla=\varphi / \varphi_{0}$, this gives

$$
F_{N}^{C}(y)=\left[\frac{3}{y^{3}}(\sin y-y \cos y)\right]^{2}
$$

which gives diffraction minima at $y=4.49,7.7$, otc. Schiff ${ }^{11}$ in particular has considered the form of $F_{\mathrm{N}}^{C}(y)$ in Born approximation for various simple analytic forms for $\rho(r)$. We note that $F_{N}^{C}$ is of the form of the square of a real amplitude term, and thus gives diffraction minima when the amplitude changes sign. For distributions sufficiently peaked in the center and with a gradual "talling-of $f$ ", the amplitude may not change slgn and 
$\mathrm{F}_{\mathrm{N}}^{\mathrm{C}}$ will be a smoothly decreasing function of $\mathrm{y}$. It is readily seen from Eq. Al that $F_{N}^{C}(0)=1$ and, by expanding the exponentials and using inversion symmetry through $r=0$, that the leading term in the decrease of $\mathrm{F}_{\mathrm{N}}^{\mathrm{C}}$ for small $\mathrm{y}$ depends on $\left\langle\mathrm{r}^{2}\right\rangle$. In this connection it is interesting to note that the widely used Williams ${ }^{I}$ formula uses $V(r)=\left(Z e^{2} / r\right)\left(I-e^{-2 r / R}\right)$ for which $\rho(r)=(4 / 0 / 3)(R / r) e^{-2 r / R}$, and $\left\langle r^{2}\right\rangle=3 R^{2} / 2$. Here $\rho_{0}$ is the charge density for a uniform nucleus of radius $R$. Although Williams implied that this distribution closely approximates a uniform model, and it has been taken by others ${ }^{6}$ as corresponding to the uniform model, it actually corresponds to a rather strongly peaked distribution about $r=0$ with $\left\langle r^{2}\right\rangle$ larger by a factor of $5 / 2$ than for a uniform distribution, and thus a correspondingly more rapid initial decrease of $F_{N}^{C}$ for small $y$. For larger y it gives a uniform decrease of $\mathrm{F}_{\mathrm{N}}^{\mathrm{C}}$ with $\mathrm{y}$ with an asymptotic form $16 / \mathrm{y}^{4}$ for large $\mathrm{y}$. In the region of large $\mathrm{y} \mathrm{Eq}$. A2, between minima, has a steady decrease with $\mathrm{y}$ which can be estimated by neglecting the sin $\mathrm{y}$ term and setting $|\cos \mathrm{y}| \approx 1$ to give an asymptotic form $9 / \mathrm{y}^{4}$, which is below that for the Williams distribution.

The detailed phase shift calculations of Yennie, Ravenhall, and Wilson, using various assumed $\rho(r)$, show that the shape of $\mathrm{F}_{\mathrm{N}}^{\mathrm{C}}(\mathrm{y})$ is energy-dependent and significantly different from the Born approximation value for high $z$ materials. In particular, the scattering amplitude is a complex number which circles the 
value zero in the complex plane when "changlng sign". Thus the "diffraction minima" are largely missing, or are greatly reduced In magnitude compared to the Born approximation results. This feature is also apparent in the experimental results and had led to an initial "Born approximation interpretation" that $\rho(r)$ must resemble an exponential distribution. The interpretation favored at the time of this writing is that $P(r)$ can be fairly constant for values of $r$ containing most of the charge distribution, with a gradual dropping off at the "surface".

In view of the above results, and with the consideration that we wish to choose a form for $\mathrm{F}_{\mathrm{N}}^{\mathrm{C}}$ which will not underestimate the expected multiple scattering due to the known electromagnetic interaction of mu-mesons with protons in examining the results of experiments investigating possible "anamolous scattering", we have chosen the following form for $\mathrm{F}_{\mathrm{N}}^{\mathrm{C}}$. For $\mathrm{y}=0,1,2,3$ we choose $\mathrm{F}_{\mathrm{N}}^{\mathrm{C}}=1.00,0.82,0.50$; and 0.15 to approximate Eq. A2 after the effect of the first diffraction minimum is "removed". A smooth curve through these points is then joined smoothly to $\mathrm{F}_{\mathrm{N}}^{\mathrm{C}}=12 / \mathrm{y}^{4}$ for $\mathrm{y} \geqslant 4$, this being between the Wililams formula and the value obtained above for a unfform distribution. In principle this should be applied to the cross section for total angle scattering rather than for projected angle scattering as we do here. The consequences of this approximation are discussed following the discussion of $\mathrm{F}_{\mathrm{c}}^{\mathrm{I}}$.

The calculation of the inelastic scattering is quite dif- 
ficult to perform exactly. Amaldi, Fidecaro, and Mariani ${ }^{I / t}$ have

14 E. Amaldi, G. FIdecaro, and F. Mariani, Nuovo Cimento I, 553 (1950)

carried out detalied calculations of the inelastic and elastic scattering expected, using a particular independent particle nuclear model. For incldent particles of very high kinetic energy and $\mathrm{cp} \sim 100 \mathrm{Mev})$, a Born approximation treatment similar to that used in the theory of $x$-ray scattering by atoms should be reasonably accurate and gives the result, as for $x$-rays, ${ }^{15}$ that

15 c. f. A. H. Compton and S. K. Allison, "X-rays in Theory and Experiment", D. Van Nostrand and Co. (1935), Chapter 3. $F_{N}^{I} \approx Z^{-I}\left(I-F_{N}^{C}\right)$ times the form factor for the scattering by a single proton. This last factor mast be included if the proton Is not effectively a point charge (due, say, to meson cloud effects). Amaldi etal ${ }^{14}$ have partlcularly emphasized this point and have calculated the expected effect of the proton "size" on the basis of a simple model. Experimentally, however, it seems that the proton charge distribution should be treated as being confined to a surprisingly small volume on the basis of electron scattering experiments, ${ }^{16}$ and, by inference, from the interpre-

$16 \mathrm{~J} . \mathrm{A}$. McIntyre and R. Hofstader, Bulletin of the American Physical Society, Vol. 29, paper Il (also see paper I2) A.P.S. meeting at seattle, Washington, July, 1954. 
tation 17 of the experiments on the neutron-electron interaction.

17 Soe L. I. Foldy, Phys. Rev. 87, 675 (1952) for a discussion of this subject and for reference to earlier papers.

The experiments show that the proton form factor is essentially unity for op $\sim 200 \mathrm{Mev}$ and at angles $\sim 90^{\circ}$, so we take it to be essentially unity for cp $\phi$ values considered in this paper. For large enough value of $c p \varphi$ this factor will eventualiy become 1mportant and require consideration.

If the single proton form factor is set equal to unlty, the above expression for $F_{N}^{I}$ can be understood by analogy with $x-r a y$ scattering where the "Inelastic" scattering corresponds to modifled Compton elastic scattering, where the recoll momentum is taken up by a single (moving) electron rather than by the atom as a whole. The final states of importance correspond to recoll electron momenta centered about the photon momentum transfer; modified by the initial electron momentum distribution, which Is given by the Fourler transform of the ground state wave function of the atom. For charged particles of kinetic energy and $c p>$ 100 Mer, and for $y>1$, the final states of 1mportance should be attalnable with moderate energy loss, so the expression for the total scattering intensity in a given direction can be written with falr accuracy by suming over-all final states, keeping $\vec{q}_{\text {, }}$ for a given angle, the same as in the elastic case.

\section{page thirty-four}




$$
\begin{aligned}
Z^{2}\left[F_{N}^{c}+F_{N}^{I}\right] & =A^{2} \sum_{f}\left|\left\langle f\left|\sum_{j} e^{-i \vec{q}_{j} \cdot \vec{\Omega}_{j}}\right| i\right\rangle\right|^{2} \\
& =A^{2}\left\langle i\left|\sum_{m} e^{i \vec{q}_{m} \cdot \vec{\Omega}_{m}} \sum_{j} e^{-i \vec{q}^{\prime} \cdot \vec{\Omega}_{j}}\right| i\right\rangle
\end{aligned}
$$

by closure. For an independent particle model Eq. Al and Eq. A3 lead to the above quoted result for $F_{N}^{I}$. Thus we set

$$
\mathcal{F}_{N}=F_{N}^{c}+F_{N}^{I}=F_{N}^{c}+Z^{-1}\left(1-F_{N}^{c}\right)
$$

The remaining point to be considered is the error introduced when $\mathcal{F}_{N}$ is applied to the law for projected (rather than total) angle scattering. For a do/d $\Omega$ law varying as $\varphi^{-n}$, the projected angle single scattering law is obtained by multiplying $d \sigma / d \Omega$ by $\varphi C(n)$, where $c(n)=\pi / 2,4 / 3,16 / 15,5 \pi / 16$, and $32 / 35$ for $n=4,5,7,8$, and 9 respectively $\left(c(n)=2 \int_{0}^{\pi / 2} \cos ^{n-2} \varphi d \varphi\right)$ In going from $n=4$ for Rutherford scattering to $n=8$, (which is obtained when the asymptotic form of $F_{N}^{C}$ multiplies the Rutherford scattering, $c(n)$ is reduced by the factor of 5/8. This represents an extreme situation since $\mathcal{F}_{\mathrm{N}}$ is more slowly varying. We note that the above effect can approximately be taken into account by choosing $r_{0} \sim 10$ percent larger than otherwise when applying $\mathcal{F}_{\mathrm{N}}$ to the law for projected angle scattering. In the examples we choose $r_{0}=1.1 \times 10^{-13} \mathrm{~cm}$, corresponding to $r_{0} \approx$ $1.0 \times 10^{-13} \mathrm{~cm}$ for $\mathrm{d} \sigma / \mathrm{d} \Omega$. In view of the uncertainty concerning the exact form for the true $\mathcal{F}_{\mathbb{N}}(y)$, we consider this approximation to be adequate for the present. 


\section{APPENDIX B. DISCUSSION OF THE EXPERIMENTS 5,6 ON MU-MESON SCATTERING}

The interest of the authors in the multiple scattering theory was mainly stimulated by the possible consequences of a strong anomolous mu-meson-nucleon interaction on the interpretation of the mu-mesonic $x$-ray experiments. ${ }^{12}$ Cosmic ray experiments on the scattering of fast mu-mesons by nuclei suggested that some enomolous scattering exists, out the interpretation of the exper1ments are not completely unambiguous, and there is considerable disagreement on the magnitude and existence of the effect. When these experiments were analysed, the expected multiple scattering distribution for an extended nucleus was obtalned by various approximations such as comparing the experimental results with the predictions of the Molière and Olbert theories, or by using the Willians theory. In all cases the old "large" nuclear size was used which gives $\left\langle r^{2}\right\rangle$ twice that favored by recent experiments. Thus the experiments were always analysed on a basis that underestimated the coulomb multiple scattering. Aside from the results of the experiments discussed belcw, we note that the mu-mesonic $x$-ray results indicate that any anomolous energy independent nuclear potential for the mu-meson can in its effect at most be equivalent to a slight change in the cholce of the nuclear radius when calculating the coulomb interaction. This could not explain any significany portion of the anomolous scattering reported in

page thirty-six 
some of the experiments. Also, experiments on the nuclear inter action by Annis et al ${ }^{18}$ can be explained without invoking any

18 M. Annis, H. C. Wilkins, and J. D. M1ller, Phys. Rev. 24, $1038(1954)$

anomolous interaction. Such an "anomolous interaction" would have to be strongly energy-dependent and thus only appear strongly at high energies to explain the low energy experiments. Amaldi and Fidecaro ${ }^{5}$ investigated the large angle scattering of fast mu-mesons in the energy bands $200 \mathrm{Mev}$ to $320 \mathrm{Mev}$ and $>320$ Mev, using a counter hodoscope. They compared the large angle multiple scattering in iron and lead, emphasizing the iron results as far as anomolous scattering is concerned. About $5 \times 10^{5}$ incident mesons were counted and results for iron were consistent with no anomolous interaction. In their series 2 run on iron 249, 168 particles were incident and 3 scattered particles were observed in each energy band. When an extra $200 \mathrm{gm} / \mathrm{cm}^{2}$ of bricks were

placed above the apparatus to decrease the number of protons etc., 204, 914 particles were incident with one scattered count in the lower energy band and none in the higher energy band. The latter numbers are about the expected values for scattered protons, etc. With that amount of filtering. They set an upper limit for "anomolous scattering" of $\sim 4.5 \times 10^{-29} \mathrm{~cm}^{2} /$ nuclean in the lower energy band and $\sim 2.3 \times 10^{-29} \mathrm{~cm}^{2} /$ nuclean in the upper energy band. These values assume isotropic scattering for the anomolous 
part.

Whittemore and Shutt investigated the multiple scattering of negative mu-mesons in $5 \mathrm{~cm}$ of lead for particles having 0.3 Bev $\leqslant$ $c p \leqslant 3.1$ Bev using two cloud chambers and a magnet for momentum analysis: Their experimental points essentially fall on the ScottSnyder (Molière) curve for $p \varphi \leqq 13$ Bev degrees, with one polnt at 17 Bev degrees a factor of about two below the Molière curve (but with a large statistical uncertainty). From Fig. 5 these points would also fall above our theoretical curve. The principle difflculty in the interpretation of these results is the question of the certainty that only mu-mesons were involved. It is of interest In this connection that the later experiments were conducted underground, or, in one case, using 1 meter of lead absorber above the cloud chamber to assure greater beam purity. Also we should like to point out that measurements ${ }^{19}$ on the scattering of cp $200 \mathrm{Mev}$

19 John 0. Kessler and Leon M. Lederman, Phys. Rev. 94, $689(1954)$

$\pi$-mesons on $\mathrm{Pb}$ show differential cross sections for elastic plus Inelastic scattering which are always $\sim 0.1$ barn or larger. By contrast, the elastic scattering experiments using electrons of comparable momentum give differential cross sections which are $\sim 10^{-6}$ barns at $120^{\circ}$, a factor of $10^{5}$ below the $\pi$-meson cross section. This contrast is admittedly extreme, but it emphasizes the importance of not underestimating the possible importance of 
small percentages of beam contamination in such experiments. The remaining experiments used greater absorber thickness at sea level, or operated underground to minimize beam contamination. They have the common feature, however, that the momentum distribution of the incident particles was not measured directly, but was assumed known from other sources. Any error in the assumed. known momentum distribution would tend to affect all of these experiments in a similar fashion. The fact that the observed scattering distributions are not given directly as a function of $p \varphi$ makes comparison of their final curves with our calculated $M(\varphi)$ difficult.

George, Redding, and Trent measured the multiple scattering of penetrating cosmic ray particles in $2 \mathrm{~cm}$ lead plates at $60 \mathrm{m.w} . \theta$. underground using a counter-triggered cloud chamber. Three experimental arrangements were used for the triggering counter telescope Involving 0,5 , and $10 \mathrm{~cm}$ lead below the cloud chamber. The particles were all assumed to be $\mu$-mesons and the momenta of the individual particles were known only to be above the cutoff values determined by the lead absorber thickness. The analysis was made by assuming that the energy distribution was flat for $E<<E_{0}=12$ Bev. The experimental distribution $N(\varphi)$ was compared with one calculated using a welghted average of gausian functions (one for each energy) in accord with the above prescription for the assumed energy distribution. No anomolous scattering was observed using $10 \mathrm{~cm}$ of lead, but a small amount of "anomolous scattering" appeared at larger angles when 0 or $5 \mathrm{~cm}$ 
lead was used. They conclude that no anomolous scattering is observed for kinetic energles > $200 \mathrm{Mev}$, and some may exlst for lower energies. It is interesting to note that the curves for 0 and $5 \mathrm{~cm}$ lead show essentlally the same excess over their theoretical curves whether point nucleus scattering is included or not. It would be desirable to have a direct measurement of the momentum distribution of the incident particles.

Leontic and Wolfendale used a multiplate cloud chamber at sea level with a counter telescope that required that detected particles traverse 1 meter of lead above the chamber, six $2 \mathrm{~cm}$ lead plates inside the chamber, and 0,5 , or $10 \mathrm{~cm}$ lead below the chamber. It was assumed that the 1 meter of lead excluded all but $\mu$-mesons from the measurements. The main analysis compared the maximum scattering in any of the center four plates with the r. m. s. angle for the four plates. The analysis made the assumption that the basic multiple scattering law for any given particle should effectively be a gaussian at all angles if no anomolous scattering were present. By an ingenious analysis they showed that the results were inconsistent with this assumption. A further analysis assumed that an "anomolous scattering gaussian" would be superimposed on the normal multiple scattering gaussian in some small fraction of the plate traversals. They then obtained a best matching of parameters. Unfortunately, the selection criterion for considered events was biased in favor of selecting cases where "anomolous scattering" (or the non-gaussian multiple scattering tail) occured. Dr. Wolfendale informs ${ }^{20}$ us that a 
proliminary ro-examination suggests that the ossential features of the results will not bo seriously altered on correcting this bias factor, but ware not seen the detalls of such a re-examination.

The andements of Loont1c and Wolfendale have been extendod by MCDiarmito using a diforent and interesting analysis.

20 Private Communleation. Wo wlsh to thank Professor G. D. Rochester and Dr. A. W. Wolfendale for corresponding with us concerning the enalysis of the experiments of the Manchester group on $\mu$-meson scattering. The paper by McDiarmid is scheduled for publication in Phil. Mag.

The results seom to contradict those of George, Redding and Trent In that no enomolous catterimg is observed for low energies. At higher energie the experimental results are between the Molière and 0lbert mitiple scattering curves for a (partialiy) assumed distribution of Incioint particle energies. The results are closer to the Mollaro than to tho olbert curves. We have not, however, performed the detalied folding together of our final curve with their derived momentum distributions as is necessary for a quant1tative comparison.

References to earlier papers will be found in the articles discussea above. 


\section{2}

FIGURE CAPTIONS

F1g. 1. Curves illustrating the first method of computing the multiple scattering distribution $M_{l}(y)$ for $c p=1$ Bov In $2 \mathrm{~cm} \mathrm{~Pb}$, including only coherent scattering. $\mathrm{g}=$ single scattering law; ${ }^{M_{A}}=$ multiplo scattering gaussian for single scatterings through $\mathrm{y} \leqslant 0.5 ; \mathrm{M}_{\mathrm{BI}}=$ multiple scattering distribution for single scatterings through $\mathrm{y}>0.5 ; \mathrm{F}_{\mathrm{N}}^{\mathrm{C}}$ is the assumed coherent nuclear form factor. Multiply y by 1.74 for Ber degrees. A nuclear size $R=1.0 A^{1 / 3} \times 10^{-13} \mathrm{~cm}$ was used for all of the examples of the figures, as discussed in Appendix A.

F1g. 2. Curves for the same case as in Fig. 1. The Molière and Olbert multiple scattering distributions are shown for comparison with $M_{I}(y)$. The Olbert distribution is for a single scattering cutoff angle of $\mathrm{y}=$ 1.0. The point nucleus and extended nucleus single scattering distributions are $g^{l}$ and $g$.

Fig. 3. Curves for $\mathrm{cp}=1 \mathrm{Bev}$ and $2 \mathrm{~cm} \mathrm{~Pb}$ using $\mathcal{f}_{\mathrm{N}}$ for the total olastic plus inelastlc scattering. $g^{\prime}=$ polnt nucleus single scattering law; $g=g ! \mathcal{F}_{N}$ is the assumed extended nucleus single scattering law; $M_{1}$ is the resulting multiple scattering distribution. Multiply y by 1.74 for Bev degrees.

Fig. 4. Curves for $\mathrm{cp}=1 \mathrm{Bev}$ and $5 \mathrm{~cm} \mathrm{~Pb}$ using only coherent scattering. $g^{1}, 8, \mathcal{F}_{N}$, and $M_{1}$ have the same meaning 
43

FIGURE CAPTIONS (Continued)

as in the proceding figures. Multiply $y$ by 1.74 for Bev degrees.

Fig. 5. Curves for $\mathrm{cp}=1 \mathrm{Bev}$ and $5 \mathrm{~cm} \mathrm{~Pb}$ using the total elastic plus inelastic scattering. The symbols have the same meaning as in the preceding figures. 


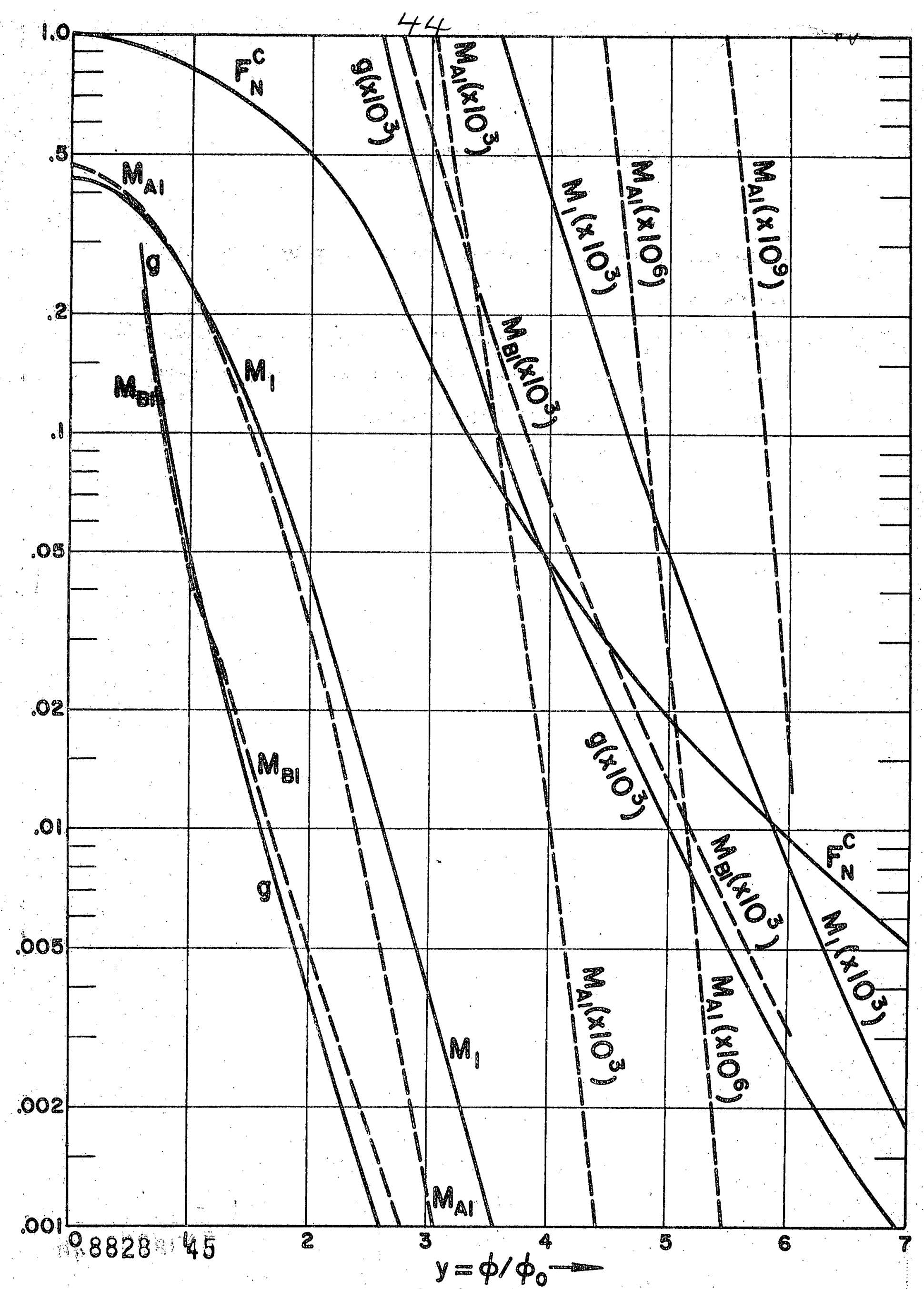




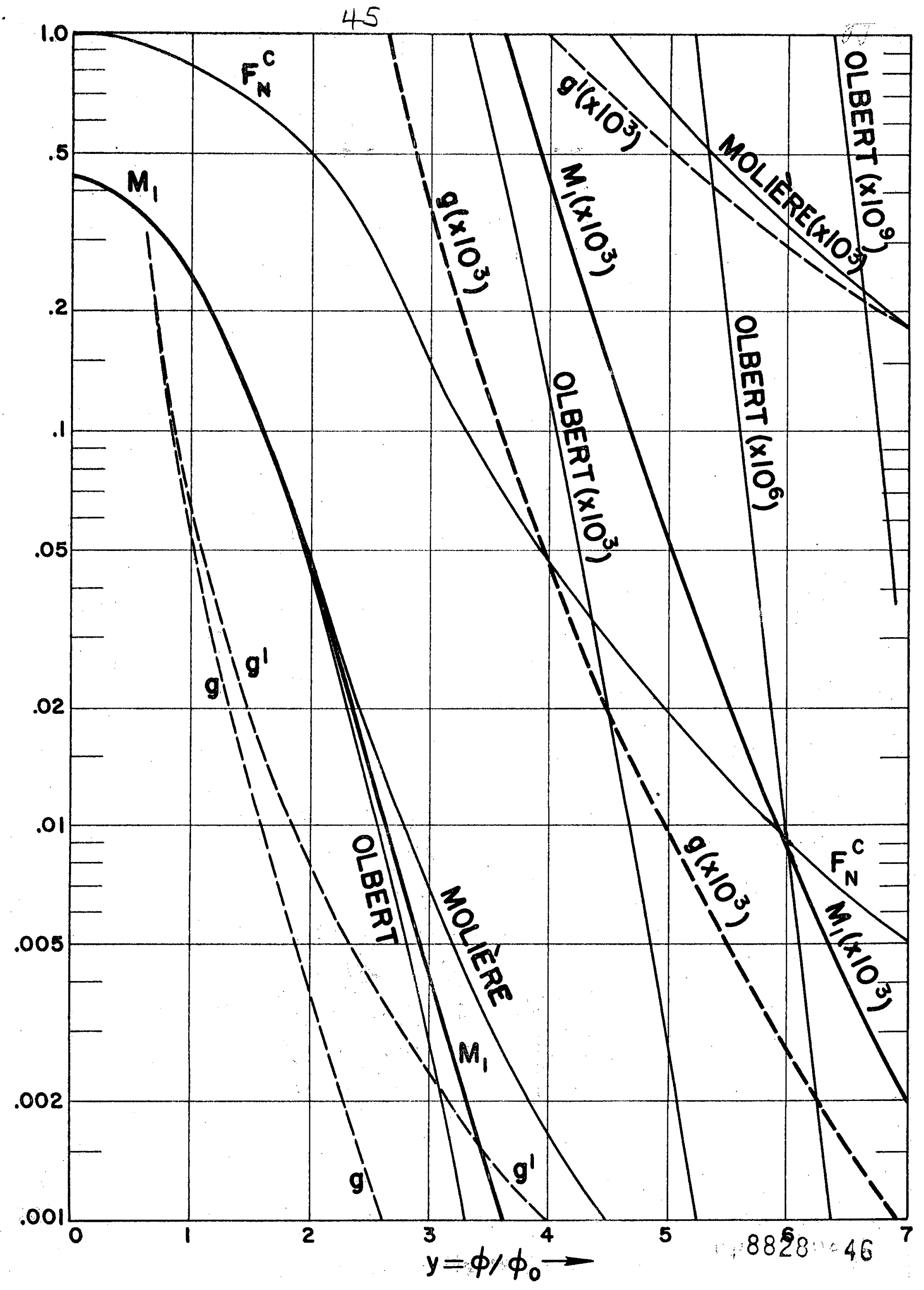




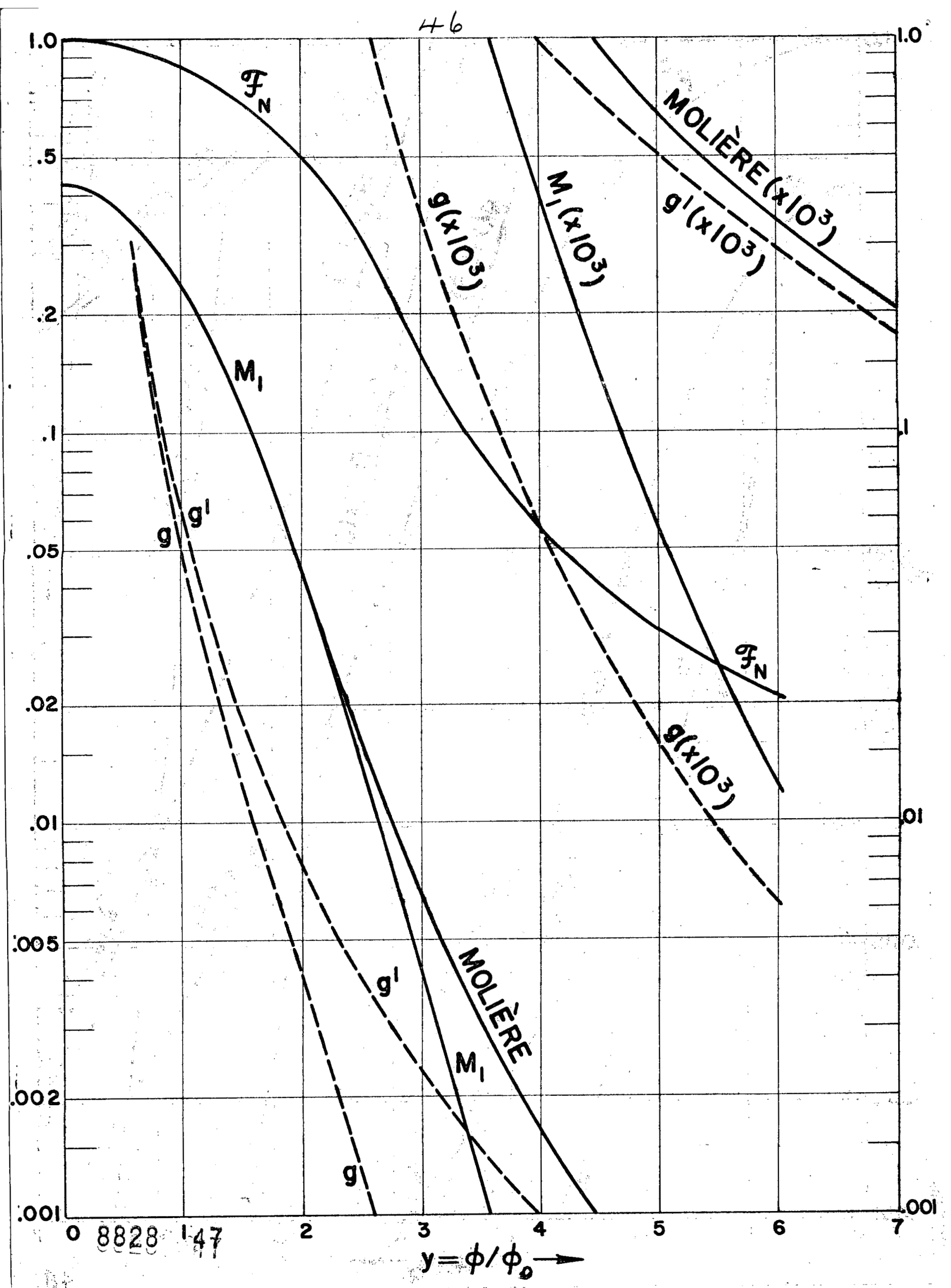




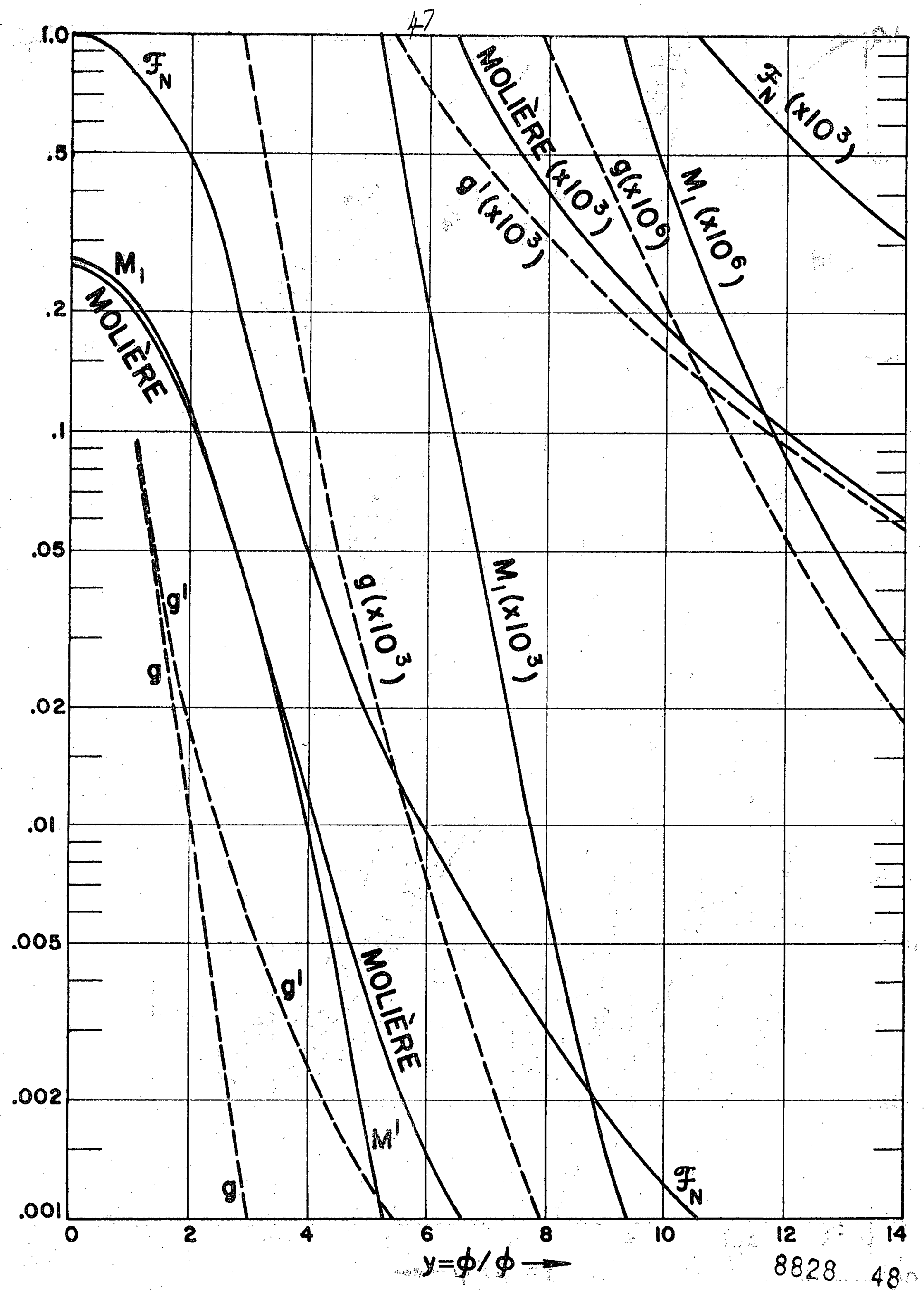


TABLE I. Values of $T(x, \lambda) \lambda^{-3}=\left[e^{\frac{49}{-(x+\lambda)^{2}}}+e^{-(x-\lambda)^{2}}-2 e^{-x^{2}}\right] \lambda^{-3}$

\begin{tabular}{|c|c|c|c|c|c|c|c|}
\hline$x=0$ & $x=1$ & $x=2$ & $x=3$ & $x=4$ & $x=5$ & $x=6$ & $x=7$ \\
\hline & & $\times 10^{-1}$ & $x 10^{-2}$ & $x 10^{-5}$ & $\times 10^{-8}$ & $\times 10^{-12}$ & \\
\hline-7.755 & 2.793 & 10.540 & 1.9112 & 3.650 & 0.8463 & 0.2504 & \\
\hline-3.539 & 1.1875 & 5.656 & 1.3507 & 3.649 & 1.2620 & 0.5793 & \\
\hline-2.040 & 0.5936 & .4 .113 & 1.4421 & 6.079 & 3.385 & 2.537 & \\
\hline-1.2642 & 0.2826 & 3.314 & 1.8069 & 12.319 & 11.251 & 13.887 & \\
\hline$x 10^{-1}$ & $x 10^{-2}$ & $x 10^{-1}$ & $x 10^{-2}$ & $\times 10^{-4}$ & $\times 10^{-6}$ & $\times 10^{-9}$ & $x 10^{-13}$ \\
\hline-8.094 & 10.751 & 2.730 & 2.382 & 2.659 & 0.3999 & 0.08138 & 0.02241 \\
\hline-5.301 & 1.3329 & 2.199 & 3.116 & 5.719 & 1.4178 & 0.4756 & 0.2159 \\
\hline-3.557 & -3.087 & 1.6845 & 3.907 & 11.810 & 4.827 & 2.670 & 1.9982 \\
\hline-2.454 & -4.597 & 1.2042 & 4.595 & 22.89 & 15.426 & 14.067 & $17 \cdot 360$ \\
\hline$x 10^{-2}$ & $\times 10^{-2}$ & $x I 0^{-2}$ & $\times 10^{-2}$ & $x 10^{-3}$ & $x 10^{-4}$ & $\times 10^{-7}$ & $\times 10^{-10}$ \\
\hline-17.447 & -4.619 & 7.926 & 5.000 & 4.106 & 0.4561 & 0.6858 & 0.13953 \\
\hline-12.775 & -4.034 & 4.7499 & 4.983 & 6.746 & 1.2355 & 3.062 & 1.0273 \\
\hline-9.612 & -3.313 & 2.564 & 4.516 & 10.079 & 3.044 & 12.438 & 6.879 \\
\hline-7.406 & -2.657 & 1.2268 & 3.703 & 13.625 & 6.784 & 45.71 & 41.68 \\
\hline
\end{tabular}




$\begin{array}{ccccccccc}\lambda & \times 10^{-2} & \times 10^{-2} & \times 10^{-3} & \times 10^{-3} & \times 10^{-2} & \times 10^{-3} & \times 10^{-5} & \times 10^{-7} \\ 3.25 & -5.826 & -2.125 & 5.039 & 27.36 & 1.6598 & 1.3625 & 1.5136 & 17.2276 \\ 3.50 & -4.665 & -1.7116 & 1.6040 & 18.159 & 1.8165 & 2.458 & 4.503 & 1.1161 \\ 3.75 & -3.793 & -1.3942 & 0.19227 & 10.800 & 1.7814 & 3.975 & 12.003 & 4.905 \\ 4.00 & -3.125 & -1.1494 & -0.28618 & 5.744 & 1.5625 & 5.748 & 28.62 & 19.283 \\ & \times 10^{-2} & \times 10^{-3} & \times 10^{-4} & \times 10^{-4} & \times 10^{-3} & \times 10^{-3} & \times 10^{-4} & \times 10^{-5} \\ 4.25 & -2.605 & -9.584 & -3.947 & 27.27 & 12.237 & 7.423 & 6.093 & 0.6769 \\ 4.50 & -2.195 & -8.074 & -3.808 & 11.539 & 8.547 & 8.547 & 11.567 & 2.118 \\ 4.75 & -1.8662 & -6.865 & -3.370 & 4.341 & 5.317 & 8.765 & 19.558 & 5.906 \\ 5.00 & -1.6000 & -5.886 & -2.921 & 1.4455 & 2.943 & 8.000 & 29.43 & 14.65 \\ & x 10^{-3} & \times 10^{-3} & \times 10^{-4} & \times 10^{-6} & \times 10^{-4} & \times 10^{-3} & \times 10^{-3} & \times 10^{-4} \\ 5.25 & -13.821 & -5.085 & -2.530 & 42.04 & 14.486 & 6.492 & 3.924 & 3.232 \\ 5.50 & -12.021 & -4.422 & -2.201 & 10.120 & 6.335 & 4.681 & 4.681 & 6.335 \\ 5.75 & -10.520 & -3.870 & -1.9268 & 1.4347 & 2.460 & 2.997 & 4.941 & 11.026 \\ 6.00 & -9.259 & -3.406 & -1.6959 & -0.5713 & 0.8479 & 1.7031 & 4.630 & 17.031\end{array}$


Tabie I (cont.)

51

\begin{tabular}{|c|c|c|c|c|c|c|c|c|}
\hline$\lambda$ & $x \geq 0^{-3}$ & $x 10^{-3}$ & $\times 10^{-4}$ & $\times 10^{-7}$ & $\times 10^{-6}$ & $x 10^{-4}$ & $\times 10^{-3}$ & $x 10^{-3}$ \\
\hline 6.25 & -8.192 & -3.014 & -1.5004 & -9.050 & 25.93 & 8.586 & 3.848 & 2.326 \\
\hline 6.50 & -7.283 & -2.679 & -1.3339 & -8.813 & 7.029 & 3.838 & 2.836 & 2.836 \\
\hline 6.75 & -6.503 & -2.392 & -1.1911 & -8.000 & 1.689 & 1.5208 & 1.8465 & 3.055 \\
\hline \multirow[t]{2}{*}{7.00} & -5.831 & -2.145 & -1.0680 & -7.193 & 0.3591 & 0.5340 & 1.0726 & 2.916 \\
\hline & $x \geq 0^{-3}$ & $x 10^{-3}$ & $x 10^{-5}$ & & $x 10^{-8}$ & $x 10^{-6}$ & $\times 10^{-4}$ & $x 10^{-3}$ \\
\hline 7.25 & -5.248 & -1.9307 & -9.613 & & 6.729 & 16.610 & 5.500 & 2.465 \\
\hline 7.50 & -4.741 & -1.7440 & -8.683 & & 1.0809 & 4.576 & 2.498 & 1.8461 \\
\hline 7.75 & -4.297 & -1.5806 & -7.869 & & 0.1195 & 1.1162 & 1.0048 & 1.2200 \\
\hline \multirow[t]{2}{*}{8.00} & -3.906 & -1.4370 & -7.155 & & ---- & 0.24103 & 0.3577 & 0.7185 \\
\hline & $x 10^{-3}$ & $x \geq 0^{-3}$ & $\times 10^{-5}$ & & & $x 10^{-9}$ & $\times 10^{-6}$ & $x] 0^{-5}$ \\
\hline .25 & -3.562 & -1.3103 & -6.524 & & & 46.07 & 11.273 & $37 \cdot 33$ \\
\hline .50 & -3.257 & -1.1980 & -5.965 & & & 7.792 & 3.143 & 17.162 \\
\hline 8.75 & -2.985 & -1.0983 & -5.468 & & & 1.1660 & 0.7756 & 6.981 \\
\hline .00 & -2.743 & -1.0092 & -5.025 & & & 0.1543 & 0.1693 & 2.512 \\
\hline
\end{tabular}


0.52

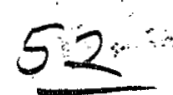

TABIE II. VALUES OF $\mathrm{g}(\mathrm{I}, \mathrm{x})$ for $\mathrm{I}=1 / 4 \cdot \quad(\mathrm{Eq}, 43)$

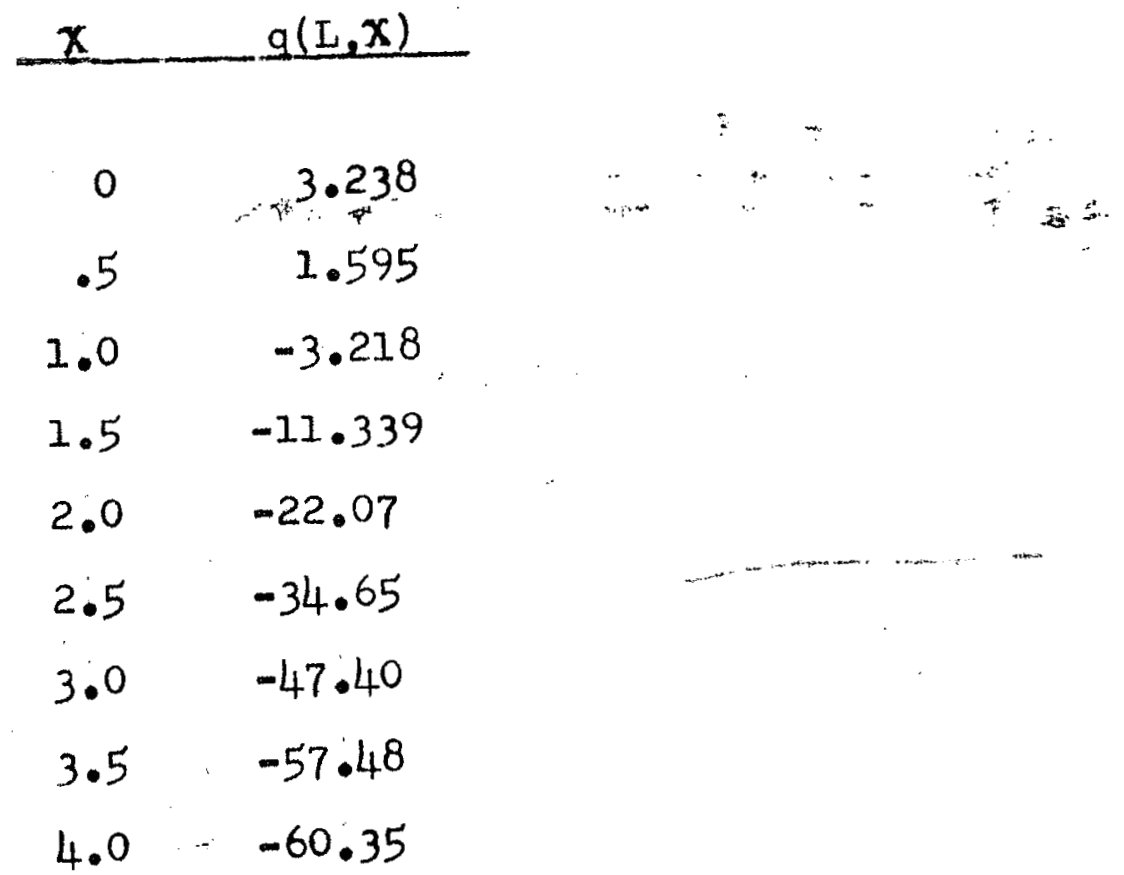

